\title{
WestVirginiaUniversity
}

THE RESEARCH REPOSITORY @ WVU

Graduate Theses, Dissertations, and Problem Reports

2016

\section{Fabrication of Nanoelectrode Arrays for Dopamine Detection}

Savan Suri

Follow this and additional works at: https://researchrepository.wvu.edu/etd

\section{Recommended Citation}

Suri, Savan, "Fabrication of Nanoelectrode Arrays for Dopamine Detection" (2016). Graduate Theses, Dissertations, and Problem Reports. 6746.

https://researchrepository.wvu.edu/etd/6746

This Thesis is protected by copyright and/or related rights. It has been brought to you by the The Research Repository @ WVU with permission from the rights-holder(s). You are free to use this Thesis in any way that is permitted by the copyright and related rights legislation that applies to your use. For other uses you must obtain permission from the rights-holder(s) directly, unless additional rights are indicated by a Creative Commons license in the record and/ or on the work itself. This Thesis has been accepted for inclusion in WVU Graduate Theses, Dissertations, and Problem Reports collection by an authorized administrator of The Research Repository @ WVU. For more information, please contact researchrepository@mail.wvu.edu. 
Fabrication of Nanoelectrode Arrays for Dopamine Detection

\author{
Savan Suri
}

Thesis submitted

to the Benjamin M. Statler College of Engineering and Mineral Resources at West Virginia University

in partial fulfillment of the requirements for the degree of

Master of Science in

Mechanical Engineering

\author{
Nianqiang Wu, Ph.D., Chair, Advisor \\ Terence Musho, Ph.D. \\ Manivannan Ayyakkannu, Ph.D.
}

Department of Mechanical and Aerospace Engineering

Morgantown, West Virginia

2016

Keywords: Nanoelectrodes, Nanosphere lithography, Electrochemistry, Diffusional domains, Dopamine

Copyright 2016 Savan Suri 


\section{Abstract \\ Fabrication of Nanoelectrode Arrays for Dopamine Detection}

\section{Savan Suri}

Recent advancements in the semiconductor fabrication technologies have greatly helped in advancing the understanding of electrochemistry at nano scale $\left(10^{-9} \mathrm{~m}\right)$. Electrodes are being produced at micro $\left(10^{-6} \mathrm{~m}\right)$ and nano scale with varied materials, designs and for diverse applications. Better electrochemical sensing and detecting capabilities are achieved with nanoelectrodes in comparison with regular macroelectrodes. Lot of theoretical studies of electrochemistry at these nanoelectrodes have been proposed and developed. Despite the theoretical advancements, little has been done in experimental studies of nanoelectrodes. The progress is majorly impeded by lack of reliable fabrication procedures to produce such nanoelectrodes and test them experimentally.

The main goal of this thesis is to develop a new procedure to fabricate nanoelectrode arrays (NEA) for enhanced electrochemical detection. A large area gold NEA is fabricated using nanosphere lithography. The electrochemical advantages of the nanoelectrodes over macro electrodes such as better mass transport of analytes, independent diffusional domains, and faster chemical reaction rates are studied. The dimensions of the electrode are optimized to get the best possible electrochemical sensing capabilities. The optimized NEA is used as a biological sensor for detecting dopamine, a neurotransmitter, in presence of biological levels of ascorbic acid.

The optimized NEA geometry has shown an excellent ability to differentiate and detect the dopamine in presence of high levels of ascorbic acid. This is attributed to the enhanced mass transport of analytes and faster chemical reaction rates at the surface of the nanoelectrodes. Bare gold macroelectrode of similar exposed area has failed to differentiate the dopamine and ascorbic acid signals. 


\section{Acknowledgement}

First and foremost, I would like to express my sincere gratitude to my advisor and mentor Dr. Nianqiang Wu for his constant support and guidance for my Master's thesis. He has been an incredible source of support and motivation showing lot of patience during my work under him. I will be always grateful for his advising.

Apart from my advisor, I would like to acknowledge and thank my Master's thesis committee members Dr. Terence Musho and Dr. Manivannan Ayyakkannu for their time, encouragement and valuable advice.

Also, my sincere thanks goes to all my lab members and the colleagues in the university: Dr. Mingjia Zhi, Dr. Scott Cushing, Dr. Jiangtian Li, Dr. Xuefei Gao, Dr. Honglei Gou, Dr. Ming Li, Dr. Fanke Meng, Peng Zheng, Joseph Bright, Jinlong Yan, Israa A Zaidan, Anveeksh Koneru, Shimeng Hao, Yang He, Sujan Kasani and Dr. Anand Kadiyala for the wonderful collaborations, knowledge sharing and fun filled times.

I would also like to express my sincere thanks to staff members of WVU Shared Research Facilities, Dr. Kolin Brown, Dr. Marcela Redigolo, Dr. Weiqiang Ding, and Harley Hart for training me on the instruments used in this work and making sure that my work moves forward with no obstacles.

Lastly, I would also express my deepest love to my respected parents, my brother, and especially my lovely wife, Nikhita Nadiminti, for supporting me unconditionally in whatever I pursue. 


\section{Table of Content}

Abstract

Acknowledgment

iii

Table of Content

iv

List of Figures

vii

List of Tables

ix

Chapter 1: Introduction

1.1 Motivation

1.2 Objectives

1.3 Significance

1.4 Thesis Organization

Chapter 2: Background \& Literature Review

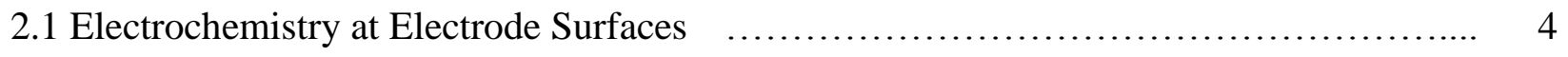

2.1.1 Faradaic Processes

2.1.2 Electric Double Layer and Charging Current

2.1.3 Mass Transport of Analytes

2.1.4 Electron Transfer Kinetics

2.1.5 Cyclic Voltammetry

2.2 Electrochemistry at Micro and Nanoelectrode Surfaces 


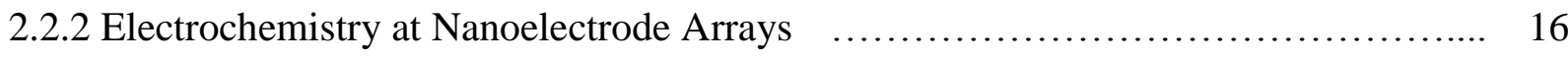

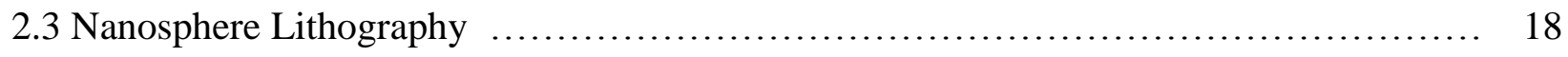

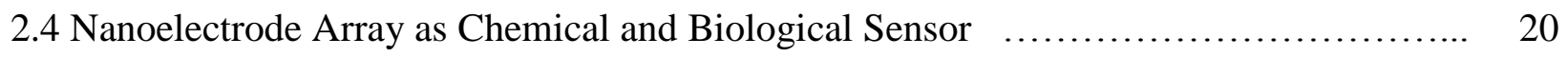

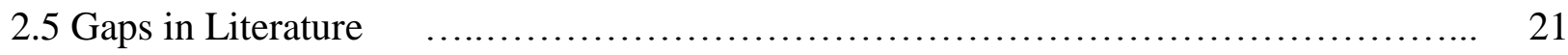

Chapter 3: Fabrication and Electrochemical Testing of Nanoelectrode Arrays using Nanosphere

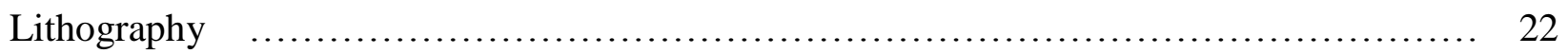

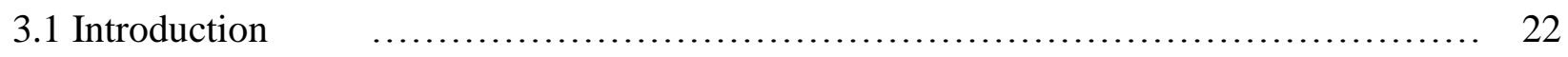

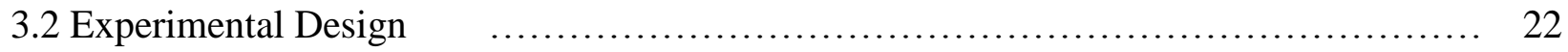

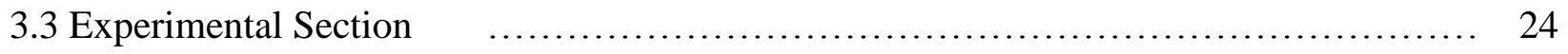

3.3.1 Materials $\quad$...................................................................... 24

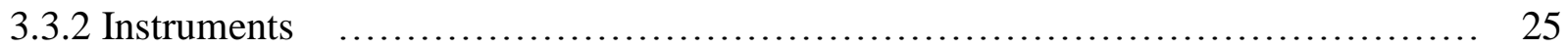

3.3.3 Fabrication of Nanoelectrodes $\quad$......................................... 26

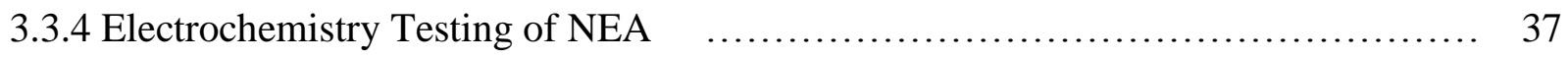

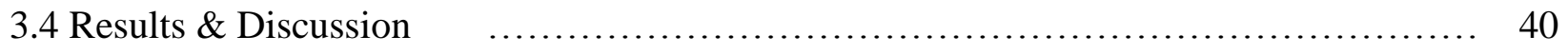

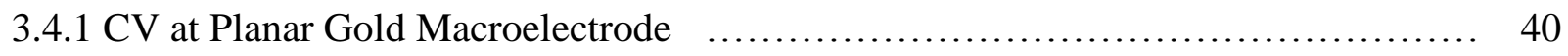

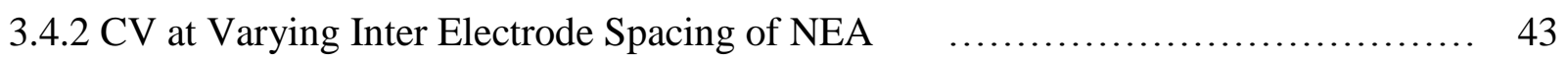

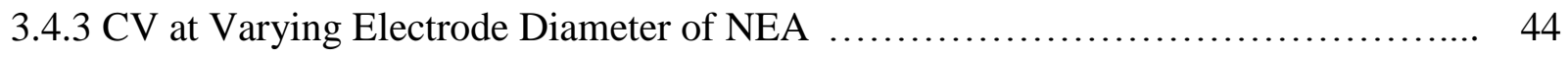

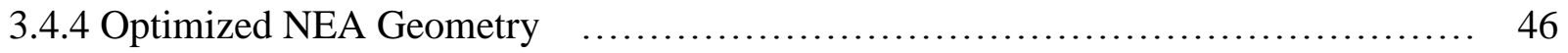


4.1 Introduction

4.2 Literature Review 


\section{List of Figures}

\section{Chapter 3}

Figure 3.1: 2 Categories of fabricated nanoelectrodes.

Figure 3.2: Simulations studies showing diffusional domain independence due to increasing

electrode spacing ${ }^{[91]}$

Figure 3.3: NEA fabrication procedure.

Figure 3.4: Closely packed monolayer of PS beads of diameter $200 \mathrm{~nm}, 500 \mathrm{~nm}$ and

$1000 \mathrm{~nm}$

Figure: 3.5: Size reduction of a $1000 \mathrm{~nm}$ diameter PS beads monolayer in an oxygen plasma asher

Figure 3.6: XPS survey scan and elemental scans of annealed XR-1541 layer

Figure 3.7: (a) XR-1541 spin speed vs annealed layer thickness and (b) Ellipsometry curves for

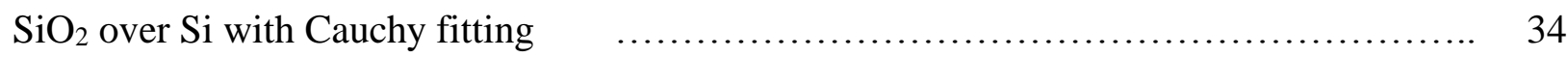

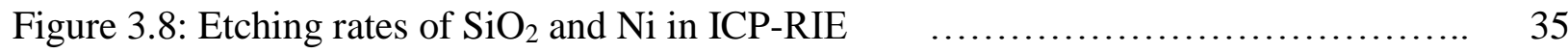

Figure 3.9: A nanoelectrode array of $\mathrm{d}=100 \mathrm{~nm}$ and $\mathrm{r}=1000 \mathrm{~nm}$

Figure 3.10: SEM images of Category 1 NEAs with a constant electrode diameter (d) of $100 \mathrm{~nm}$ and varying periodicity (r) of $200 \mathrm{~nm}, 500 \mathrm{~nm}$ and $1000 \mathrm{~nm}$.

Figure 3.11: SEM images of Category 2 NEAs with a constant periodicity (d) of $1000 \mathrm{~nm}$ and varying electrode diameter (d) of $100 \mathrm{~nm}, 400 \mathrm{~nm}$ and $700 \mathrm{~nm}$.

Figure 3.12: (a) Edge insulation for electrodes and (b) Exposed gold layer at the electrode edges.

Figure 3.13: Cell set up for the electrochemical analysis. 38

Figure 3.14: (a) Testing of the insulation layer and (b) $\mathrm{H}_{2} \mathrm{SO}_{4}$ activation of the electrodes..... 40 
Figure 3.15: (a) CV plots and (b) linear fit for square root of scan rate vs peak currents plot for the planar bare gold macroelectrode.

Figure 3.16: Comparative plots of NEAs with varying inter electrode spacing (x) at scan rates of $10 \mathrm{mV} / \mathrm{s}, 25 \mathrm{mV} / \mathrm{s}, 50 \mathrm{mV} / \mathrm{s}, 100 \mathrm{mV} / \mathrm{s}$ and $500 \mathrm{mV} / \mathrm{s} . \quad \ldots \ldots \ldots \ldots \ldots \ldots \ldots \ldots \ldots \ldots \ldots \ldots$

Figure 3.17: Comparative plots of NEAs with varying electrode diameter (d) at scan rates of 10

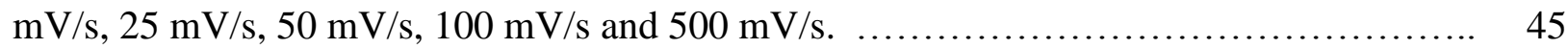

Figure 3.18: Scan rate variation plot of NEA with $d=100 \mathrm{~nm}$ and $\mathrm{x}=1000 \mathrm{~nm} . \quad \ldots \ldots \ldots$

Figure 3.19: An image of diffusion profiles at inlaid and recessed electrodes [11]. $\ldots \ldots \ldots . .48$ Figure 3.20: Peak current vs square root of the scan rate plot of NEA and planar bare gold electrode.

\section{Chapter 4:}

Figure 4.1: (a) DPV plots of NEA in PBS only and (b) DPV plot of NEA in increasing concentrations of ascorbic acid $(10 \mu \mathrm{M}, 50 \mu \mathrm{M}$ and $500 \mu \mathrm{M})$ in PBS.

Figure 4.2: Concentration plots of (a) planar gold macroelectrode with increasing concentrations of DA from $100 \mathrm{nM}$ to $50 \mu \mathrm{M}$ in $500 \mu \mathrm{M}$ AA and (b) NEA with increasing concentrations of DA from $100 \mathrm{nM}$ to $200 \mu \mathrm{M}$ in $500 \mu \mathrm{M}$ AA.

Figure 4.3: DA concentration vs. peak current plot for DPV of NEA.

Figure 4.4: Repeatability test of NEA in $500 \mathrm{nM}, 5 \mu \mathrm{M}$ and $50 \mu \mathrm{M}$ DA in $500 \mu \mathrm{M}$ AA in PBS. 


\section{List of Tables}

Table 1: CV parameters of the planar macroelectrode

Table 2: CV parameters of NEA 


\section{Chapter 1: Introduction}

\subsection{Motivation}

Recent developments in microfabrication technology and advancements in understanding of electrochemistry at nanoscale has greatly pushed the limits of electrochemical sensing and detection for varied applications. Electrodes fabricated at micro $\left(10^{-6} \mathrm{~m}\right)$ and nano $\left(10^{-9} \mathrm{~m}\right)$ scale are being extensively utilized to achieve faster analysis, extremely small detection levels and better accuracy in various environments. In 1981, Mark R. Wightman published one of the first papers shedding light on the electrochemical behavior of electrodes at micro scale ${ }^{[1]}$. The paper reviews the advantageous properties of microelectrodes over macroelectrodes such as better diffusional properties, higher chemical rate constants, easier instrumentations and its potential biological applications. The decade following the Wightman paper, lot of attention has been drawn to understand the fabrication techniques of such micro and nanoelectrodes and improve the analytical capabilities of microelectrodes for sensing and detection ${ }^{[2-4]}$.

An incredible growth of semiconductor fabrication technologies in past few decades has opened up new possibilities of fabricating and characterizing ultra small electrodes. Microelectrodes with different materials, shapes and dimensions are fabricated and studied for varied applications in different environments, not possible a few decades ago ${ }^{[5-7]}$. Despite an exponential increase in study of microelectrodes little has been done to study the fabrication and understanding of electrochemistry with nanoelectrodes. The progress is profoundly hindered by lack of large scale, easy and reliable techniques for fabricating nanoelectrodes. In this thesis, a new fabrication method is developed to produce a large area nanoelectrode array (NEA) and its electrochemical behavior 
is shown superior to a macroelectrode. The optimized NEA geometry is used to detect dopamine in presence of ascorbic acid to study the enhanced sensing capabilities of nanoelectrodes.

\subsection{Objectives}

The main aim of this thesis is to develop a new method for fabrication of nanoelectrode arrays (NEAs). This is achieved by changing the electrode sizes and distance between the electrodes. The electrochemical behavior of the NEA is investigated and the obtained results are compared with results from planar gold macroelectrode. Later, optimized NEA is used as a biosensor for biological detection. Below shows the topics to be studied and discussed in this thesis.

1) Develop a process to fabricate NEAs using Nanosphere Lithography (NSL). Nanosphere lithography is a cheap, easy and high throughput nano fabrication technique utilizing polystyrene spheres, in the order of $10^{-9} \mathrm{~m}$, to fabricate nanoelectrodes ${ }^{[8-10]}$. Electrode diameter and distance between the electrodes are tailored with changing the dimensions of polystyrene sphere as the template mask.

2) Investigate and compare the electrochemical behavior between the gold nanoelectrode array and bare gold macroelectrode. Varying electrochemical behavior is also studied with varying electrode dimensions of NEA to find the best possible design for enhanced electrochemical detection.

3) Electrochemical advantages of a nanoelectrode array over a macroelectrode is exploited to carry an ultra-sensitive biological detection. Using the optimized NEA, an electrochemical detection of dopamine, an important neuro transmitter in human brain, in presence of high levels of ascorbic acid is studied. This analysis is focused mainly to show the advantages of NEA over bare gold macroelectrodes for biological sensing applications. 


\subsection{Significance}

The study will focus on following important points.

1) A new NEA fabrication method is developed to overcome the current technical barrier for fabrication of NEA's.

2) The correlation between varying the nanoelectrode dimensions and the electrochemical properties is established, which provides the guidelines for designing better new nanoelectrodes for enhanced electrochemical activity.

3) A highly sensitive and selective electrochemical probing is realized by optimizing detection condition, leading to robust and highly capable sensors for environmental observation, biological monitoring and industrial quality control.

\subsection{Thesis Outline}

This thesis consists of 5 chapters. The first chapter primarily focuses on a general introduction to the subject of the thesis. The second chapter introduces the background and general literature review on theory and advancements made in the subject of the thesis. The third chapter involves discussion on the fabrication process of the gold NEA and a discussion on NEA's electrochemical advantage over bare gold macroelectrode. The fourth chapter is to use the fabricated nano electrode array as a biological sensor to show its advantages over bare gold macroelectrode for detection of dopamine in presence of high levels of ascorbic acid. The sixth chapter is the summary and conclusions of the work presented and a brief prospective on future advancements of the current work. 


\section{Background and Literature Review}

\subsection{Electrochemistry at Electrode Surfaces}

When an electrode is placed in an electrolyte, the electrode surface acts as a junction between an electrolyte (ionic conductor) and the electrode (electronic conductor). All the electrochemical processes take place at this electrode-electrolyte interface ${ }^{[11]}$. Usually any electrochemical system has a working electrode, a counter electrode and a reference electrode. The working electrode responds to the target analyte and it is where the signal is produced. The reference electrode is always held at a constant potential which acts as a reference potential and is independent of the properties of the solution. Third is the counter electrode which is used to complete the electrical circuit for the electrochemical analysis. The potential of the working electrode is controlled with respect to the reference electrode such as saturated calomel electrode (SCE) or silver/silver chloride $(\mathrm{Ag} / \mathrm{AgCl})$ electrode. In most of the electrochemical measurements, an external potential is applied between the working and counter electrode which is used to drive an electron transfer reaction and the resultant current is measured. The measured current reflects the rate at which the ions are transported from the solution bulk to the electrode surface which is called mass transport and the electron movement across the electrode-electrolyte interface which is called electron transfer kinetics. Thus any chemical species which is electroactive (i.e. which can be oxidized or reduced) can be measured using various electroanalytical techniques.

The measured electrical signal from various electroanalytical techniques are used for qualification and quantification of the electrochemical processes. The advantages of electrochemical analysis over other techniques such as optical and chromatographic procedures include high sensitivity and selectivity towards electroactive species, a wide linear range of detection, very small sample 
volumes, low-cost instrumentation, and usage of wide range of electrodes which can be used in unusual environments.

\subsubsection{Faradaic Processes}

The concentration of the target analytes can be measured in any potential controlled electrochemical technique by monitoring the properties of electron transfer of the redox reactions such as

$$
\mathrm{O}+\text { ne } \Leftrightarrow \mathrm{R}
$$

where $\mathrm{O}$ and $\mathrm{R}$ are the oxidized and reduced forms, respectively, of the redox couple. Such a reaction occurs in a potential window at which the electron transfer, between the ions in the solution and electrode, is thermodynamically or kinetically favorable. For any system restrained by thermodynamic laws, the concentration of the electroactive species can be established using the potential of the electrode by the Nernst equation ${ }^{[12]}$ :

$$
E=E^{o}+\frac{2.3 R T}{n F} \log \frac{C_{O}(0, t)}{C_{R}(0, t)}
$$

where $\mathrm{E}^{\circ}$ is the standard potential for the redox reaction, $\mathrm{R}$ is the universal gas constant $\left(8.314 \mathrm{JK}^{-}\right.$ ${ }^{1} \mathrm{~mol}^{-1}$ ), $\mathrm{T}$ is temperature in Kelvin, $\mathrm{n}$ is the number of electron transferred in the reaction, $\mathrm{F}$ is the Faraday constant $\left(96,487\right.$ coulombs). $C_{O}$ and $C_{R}$ are the concentrations of oxidized and reduced species respectively. This change in oxidation state of the electroactive species results in current which is termed as faradaic current as it obeys Faraday's law i.e., the reaction of 1 mol of substance involves a change of $\mathrm{n} \times 96,487 \mathrm{C}^{[12]}$. This faradaic current produced in electrochemical reaction is the direct measure of the rate of the redox reaction. The resulting current vs potential diagram, called voltammogram, displays a current signal (y-axis) and applied potential (x-axis). 
The processes involved in the reaction at the electrode surface dictates the shape and magnitude of the voltammetric response. The total current produced in any electrochemical experiment is the summation of the faradaic currents due to the reaction and a non-faradaic charging background current.

The rate of redox reaction is determined by the slowest step in the process. Simple electrochemical reactions involve mass transport of the analyte species to the electrode surface from the bulk solution, electron transfer across the electrode-electrolyte interface, and the transport of the products back into the bulk solution. The rate of the reaction, can be restrained by either mass transport of the electroactive species or the rate of electron transfer. The rate determining step of the reaction will be the slowest step in the process. The current generated is called mass-transport limited when reaction rate is controlled by rate at which the electroactive species reach the surface and the electron transfer at the electrode surface is assumed to be fast ${ }^{[12]}$. Such reactions are also known as reversible or Nernstian reactions, as they obey laws of thermodynamics.

Usually the faradaic currents or reaction rates are governed by the rates of the process such as ${ }^{[11]}$

1) Mass transport of analytes from the bulk solution to the electrode surface.

2) Electron transfer rate between the analyte (in the electrolyte) and the electrode at the electrode surface.

3) Chemical reaction before or after the electron transfer near the electrode surface.

4) Other surface reaction such as adsorption, desorption or crystallization etc. 


\subsubsection{Electric Double Layer and Charging Current}

An electrical double layer is formed at the electrode-electrolyte interface when an electrode is dipped into electrolyte. The ions of the opposite charge (in the electrolyte) are attracted towards the electrode surface to compensate the excess charge on it. In the two layers, the layer closer to the electrode surface is called inner layer or Helmholtz layer containing the solvent molecules and specifically/nonspecifically adsorbed ions onto the surface ${ }^{[11]}$. These ions are strongly held by the electrode surface by long range coulombic forces. The outer layer also called as diffuse layer is a three-dimensional region which extends into solution bulk and its thickness depends on ionic concentration in the solution. This layer reflects a balancing act between ordering forces from electrical field (from electrode surface) and the random thermal motion disorder of the ions in the solution. The equilibrium between these effects dictates the ionic concentration near the surface of the electrode. On overall, the total net charge of the two layers is equal and opposite to the net charge on the surface of the electrode.

The electrical double layer at the surface of electrode is similar to a parallel plate capacitor. The capacitance at the double layer is equal to capacitance of the inner layer and capacitance from outer diffuse layer connected in series. An abrupt drop in the capacitance is observed with dilute solutions indicating the contributions from diffuse layer. The charging in the double layer at the electrode-solution interface is responsible for the residual current or background current known as charging current in all of the electrochemical analysis techniques ${ }^{[12]}$. This type of current is nonfaradaic because electrons are not transferred across the electrode-solution interface. Charging current in the electrochemical experiments interferes with the faradaic signal and limits the detectability of the analyte. 
The residual charging current at a solid electrode is ${ }^{[12]}$ :

$$
i=C_{d l} A \frac{d E}{d t}+C_{d l}\left(E-E_{z c}\right) \frac{d A}{d t}+A\left(E-E_{z c}\right) \frac{d C_{d l}}{d t}
$$

where $C_{\mathrm{dl}}$ is capacitance per unit area, $\mathrm{A}$ is area of the electrode, $\mathrm{E}_{\mathrm{pzc}}$ is the potential at zero charge where no net charge exists in the double layer. The above equation can be used for calculating the double layer capacitance for solid electrodes. For experiments such as chronoamperometry, the charging current resembles potential applied to a series RC circuit ${ }^{[12]}$.

$$
i=\frac{E}{R_{S}} e^{-t / R C_{d l}}
$$

where $R_{S}$ is the uncompensated resistance in the electrolyte. The equation clearly shows that the current decreases exponentially with the time. At very low concentrations of analyte, the charging current can be much larger than the faradaic current. Hence, it is very important to find ways to reduce charging currents to enhance the detection limits of analytes in electrochemical experiments.

\subsubsection{Mass Transport of Analytes}

Mass transport in any electrochemical analysis occurs in three modes, i.e. diffusion, convection and migration. Diffusion of the electroactive species is caused under the influence of concentration gradient, from regions of higher concentrations to lower concentrations, trying to minimize the concentration difference. In convection, the transport of electroactive species to the electrode surface is caused by an external driving force such as mechanical stirring, flowing of the solution,

rotation or vibration of the electrode. Convection can also occur naturally due to the density gradient. Migration is the movement of charged particles along an applied external electrical field. 
The rate of mass transport at a fixed point is measured by the flux $\mathrm{J}$ which is defined as the number of molecules penetrating into an imaginary plane with a unit area, unit time and with units molcm ${ }^{-2} \mathrm{~s}^{-1}$. Mathematically the flux of electroactive species to the electrode is described by Nernst-Planck equation [12],

$$
J(x, t)=-D \frac{d C(x, t)}{d x}-\frac{z F D C}{R T}-\frac{d \phi(x, t)}{d x}+c(x, t) V(x, t)
$$

where $\mathrm{D}$ is the diffusion coefficient $\left(\mathrm{cm}^{2} / \mathrm{s}\right), \mathrm{dC}(\mathrm{x}, \mathrm{t}) / \mathrm{dx}$ is the concentration gradient, $\mathrm{d} \phi(\mathrm{x}, \mathrm{t}) / \mathrm{dx}$ is the potential gradient, $\mathrm{z}$ and $\mathrm{C}$ are the charge and concentration of electroactive species, respectively, and $\mathrm{V}(\mathrm{x}, \mathrm{t})$ is the hydrodynamic velocity (in the $\mathrm{x}$ direction). This equation involves all the three modes of mass transport i.e. diffusion, convection and migration. The current is directly proportional to the flux (A) and the surface area of the electrode (A) ${ }^{[11]}$ :

$$
i=-n F A J
$$

The situation becomes quite complex when all the three modes of mass transport are considered. The complexity can be greatly reduced by suppressing the analyte migration by adding large amounts of inert salts. An addition of a high concentration of the supporting electrolyte increases the solution conductivity and reduces the electrical fields. Usually salts such as sodium chloride $(\mathrm{NaCl})$, potassium chloride $(\mathrm{KCl})$ and phosphate buffer saline $(\mathrm{PBS})$ are used as supporting electrolytes. Although, care should be taken as the supporting electrolytes can bring some serious interference such as producing characteristic faradaic responses, reacting with the products or adsorbing on to the surface of the electrode and changing the electron transfer kinetics at the electrode. Convection effects can be completely removed by any physical motion of the electrode or the electrolyte. The reaction at the surface of the electrode creates a concentration gradient 
leading to a diffusional flux of analytes. According to Fick's first law, the rate of diffusion of the analytes in a solution is directly proportional to the slope of the concentration gradient ${ }^{[12]}$.

$$
J(x, t)=-D \frac{d C(x, t)}{d x}
$$

From equations (2.6) and (2.7), a general expression for the current response is derived [12],

$$
i=n F A D \frac{d C(x, t)}{d x}
$$

Hence, the current is proportional to the concentration gradient of the analytes and is time dependent. Such a relationship is described by the Fick's second law ${ }^{[12]}$ :

$$
\frac{d C(x, t)}{d t}=D \frac{d^{2} C(x, t)}{d x^{2}}
$$

The above equation reflects the rate of change of the analyte concentration between imaginary parallel planes at distance $x$ and $(x+d x)$ from the electrode surface. Fick's second law is appropriate for the conditions where the diffusion is linear. On the whole, the Fick's laws describe the flux and the concentration of the analytes as function of position and time.

In a quiescent solution, when an analyte is reduced or oxidized at the surface of the electrode, a concentration gradient is established near the electrode surface. There is a depletion of the bulk analyte concentration and a depletion layer is formed which is also called diffusion layer. The length of the diffusion layer is denoted by $\delta$. Initially the concentration gradient is large. As the time increases, the diffusion layer expands and concentration gradient decreases. By deriving the solutions to the Fick's law for linear diffusion a current equation is derived ${ }^{[12]}$ :

$$
i=n F A D C /(\pi D t)^{\frac{1}{2}}
$$


where $\mathrm{D}$ is the diffusion coefficient of the analyte, $\mathrm{C}$ is bulk concentration of the analyte, $\mathrm{t}$ is time of the experiment and $(\pi \mathrm{Dt})^{1 / 2}$ represents diffusion-layer thickness. This equation is called Cottrel equation ${ }^{[11]}$. The equation governs the mass diffusion transport properties of an analyte to the surface of a planar electrode. According to the equation, the current reduces with respect to the square root of the time. Based on the above equation the current at a spherical electrode is ${ }^{[11]}$ :

$$
i=n F A D C /(\pi D t)^{\frac{1}{2}}+n F A D C / r
$$

where $\mathrm{r}$ is the radius of the spherical electrode. The current response of a spherical electrode contains a time dependent and time independent terms representing the planar and radial diffusion fields respectively. So the shape of the electrode strongly changes the diffusional properties of the analyte. This unique property is exploited in electrochemistry with micro and nanoelectrodes.

\subsubsection{Electron Transfer Kinetics}

In the experiments where reaction rate (hence the current) is controlled by rate of the electron transfer, the mass transport of the analyte is assumed to be predominantly fast. In any electrochemical experiment, the actual electron transfer happens between the conduction band of the electrode and molecular orbital of the analyte. For the oxidation of a reduced analyte, the energy of the electrons in the orbital of the analyte must be equal to or higher than the energy of electrons in the conduction band of electrode. Similarly, for the reduction of an oxidized analyte, the energy of the electrons of the analyte should be lower than the energy of the electrons in the

electrode. The current-potential relationship for the reactions controlled by electron transfer rate is described by the Butler-Volmer equation ${ }^{[11]}$ :

$i=n F A k^{o}\left\{C_{o}(0, t) \exp \left[-\alpha n F\left(E-E^{o}\right) / R T\right]-C_{R}(0, t) \exp \left[(1-\alpha) n F\left(E-E^{o}\right) / R T\right]\right\}$ 
where the $\mathrm{k}^{\mathrm{o}}$ is the standard heterogeneous rate constant, $\mathrm{C}_{\mathrm{O}}$ and $\mathrm{C}_{\mathrm{R}}$ are the oxidized and reduced analyte concentrations respectively and $\alpha$ is the transfer coefficient. Rate constant for a heterogeneous electron transfer reaction is the function of applied potential and $\mathrm{E}-\mathrm{E}^{\mathrm{o}}$ is called the overpotential which is an additional potential beyond the thermodynamic requirement needed to drive the reaction. This equation implies that the net current is the difference between the currents from the forward and backward reactions. Also the net current depends on the oxidation potential and surface concentration of each form of the analyte.

\subsubsection{Cyclic Voltammetry}

Cyclic voltammetry is one of the most widely used electrochemical techniques for acquiring and understanding qualitative information such as thermodynamics of redox processes and the kinetics of electron transfer reactions. It serves as an instant method to find redox potentials of the electroactive species. Using a triangular potential waveform, the current resulting from the applied potential sweep is measured resulting in a current-potential plot called cyclic voltammogram ${ }^{[11]}$. The characteristic peaks in a cyclic voltammogram are caused by the variation of diffusion layer near the electrode surface. The resultant current traces the continuous change of the analyte concentration gradient at the electrode-electrolyte interface. The increase in the peak current corresponds to the diffusional mass transport and the current drop beyond the peak exhibits a $\mathrm{t}^{-1 / 2}$ dependence which is independent of applied potential. For similar reasons, the reversal current has the same shape as the forward current.

The peak current in the reversible couple is given by Randles-Sevcik equation ${ }^{[12]}$ :

$$
i_{p}=\left(2.69 \times 10^{5}\right) n^{\frac{3}{2}} A C D^{\frac{1}{2}} v^{\frac{1}{2}}
$$


where $\mathrm{n}$ is the number of electrons, $\mathrm{A}$ is the electrode area, $\mathrm{C}$ is the analyte concentration, $\mathrm{D}$ is the diffusion coefficient, and $v$ is potential scan rate. The current density $\left(i_{d}\right)$ is directly proportional to concentration of the analyte and increases with the square root of the scan rate. Dependence of the current on the scan rate suggests that the reaction is controlled by mass transport. For a simple reversible analyte couple, the ratio of the forward current peak to reverse current peak is one. The forward and reverse current peaks are deduced from preceding baseline current. The separation between the forward and reverse peak potentials is given by ${ }^{[11]}$ :

$$
\Delta E_{p}=E_{p, f}-E_{p, r}=\frac{0.059}{n} V
$$

The peak separation potential can be used to determine the number of electrons transferred in a reaction and is a criterion for Nernstian behavior. A fast one-electron process exhibits a $\Delta \mathrm{E}_{\mathrm{p}}$ of about $59 \mathrm{mV}$. Both the cathodic and anodic potentials are independent of the scan rate.

For irreversible electrochemical processes, where the electron exchange is slow, the forward and reverse peak currents are reduced. The peak positions are shifted and separated widely. For quasireversible systems, the current is controlled by both mass transport and charge transfer. The current-potential curves of a quasi-reversible systems are also shifted and display a large separation in the peak potentials compared to a reversible system.

\subsection{Electrochemistry at Micro and Nanoelectrode Surfaces}

Advancements made in electronics industry, esp. in 1980s, made it clear that many new areas can be explored with miniaturizing the electrodes that were not accessible with larger electrodes ${ }^{\text {[13-15] }}$. In the recent times, the substantial work on micro and nanoelectrodes has offered several attractive and important properties that have expanded the possibilities of electrochemical analysis ${ }^{[16-21]}$. The term nanoelectrodes is commonly referred to the electrodes with at least one dimension not 
greater than $1 \mu \mathrm{m}$. Electrodes at such small dimensions have many practical advantages such as measurement of local concentration profiles, detection in microflow systems, analysis of very small quantities of sample and analyzing the analytes at microscopic surface areas such a biological cells. Below mentioned are some of the important properties of the micro and nanoelectrodes leading to a high signal to noise ratio.

1) As microelectrodes and nanoelectrodes result in very small total currents, it is possible to work in highly resistive solutions that would develop large ohmic (iR) drop with macroelectrodes. This allows the nanoelectrodes to be used for electrochemical measurements with very little or with no added supporting electrolytes helping greatly in extending the electrochemical potential window of the analysis ${ }^{[11]}$.

2) The smaller area of microelectrodes and nanoelectrodes greatly reduces the double layer capacitance resulting in electrochemical cells with small RC time constants. The small RC constants allow high speed voltammetric experiments to be performed at a very small timescale. And hence to probe the kinetics of very fast electron transfer and coupling reactions ${ }^{[1]}$.

3) Enhanced rates of mass transport to electroactive species due to the radial (non planar) diffusion to the edges of the nanoelectrodes. Edge effects contribute significantly to the overall diffusion current. The rate of mass transport to and from the electrode increases as the electrode size decreases. Hence, the current density increases. As a consequence, of the increase in mass transport and reduced charging current, nanoelectrodes exhibit excellent signal to noise ratio in comparison with macro electrodes ${ }^{[11]}$. 


\subsubsection{Diffusion at Nanoelectrodes}

The total diffusion limited current is sum of the planar flux and radial flux diffusion components [11]:

$$
i_{\text {total }}=i_{\text {planar }}+i_{\text {radial }}
$$

For the disk electrodes, the general expression for the radial component is given by [11]:

$$
i_{\text {radial }}=4 r n F D C
$$

where $\mathrm{r}$ is radius of the electrode, $\mathrm{n}$ is number of electrons involved in the reaction, $\mathrm{F}$ is faraday constant, $\mathrm{D}$ is diffusivity of the analyte and $\mathrm{C}$ is the concentration of the analyte in the electrolyte. Radial diffusion at the perimeter of the electrode leads to a larger flux than at the center where linear diffusion dominates. The relative dimensions of the electrode and diffusion layer will dictate the extent to which the planar and radial components dominate. If the diffusion layer thickness is larger than size the electrode the current approaches a steady state and a sigmoidal voltammogram is observed. This is due to better mass transport of electroactive species to the electrode due to dominating radial diffusion leading to steady supply of electroactive species. In contrast, if the diffusion layer is smaller than the electrode size, planar diffusion dominates and a peak shape voltammogram is observed. This is due to reduction in faradaic current due to depleting supply of electroactive species at the electrode surface. Hence, depending on the scan rate of analysis the voltammogram shape may be a peak shaped or sigmoidal. With nanoelectrodes, in chronoamperometric experiments, a steady state current is achieved rapidly after the potential step. 


\subsubsection{Electrochemistry at Nanoelectrode Arrays}

An individual nanoelectrode produces a very low signal. To amplify the signal by keeping the properties of the nanoelectrodes intact, they are added in a parallel configuration with insulator as a separation layer and aggregate all the signals from each nanoelectrode to produce a summed up signal. One example of such geometry is a nanoelectrode array. If nanoelectrode are assembled in a disordered fashion, they are called nanoelectrode ensemble (NEE). If nanoelectrodes are placed in an ordered fashion by controlling the distance between the electrodes, they are called nanoelectrode array (NEA). The distance between the nanoelectrodes greatly influences the diffusion layers of individual electrodes. As long as there is a negligible overlap of the diffusion layers from the adjacent sites, i.e. each electrode maintains its own radial diffusional field, the current of the electrode array is the sum of the currents of the individual sites maintaining a steady state current. When electrode meets insulator at the edge of the electrode, the diffusion is effectively to a point. Therefore, the diffusion profile becomes convergent and rate of mass transfer is large. This is called edge effect ${ }^{[12]}$. So smaller the nanoelectrode surface area higher the edge effect leading to higher mass transport of the analyte. Also, this enhanced rate of mass transport due to convergence leads to the steady state signal characterized by sigmoidal voltammograms ${ }^{[13]}$.

After adequately long time, the diffusional domains expand and overlap causing the nanoelectrodes to behave as if the entire geometric surface was active. Therefore, larger interspacing distance and smaller electrode sizes are preferred. Smaller interspacing distance cause the diffusion domains to overlap and leading to a planar diffusion wave. Electrodes with a very large spacing reduce the effective coverage of the diffusional activity. Hence, optimum electrode distance spacing is determined to prevent overlapping of individual diffusion layers and get highest electrode density possible leading to better detecting capabilities of the sensor. In addition to large 
current densities, the nanoelectrode array has enhanced signal to noise ratio and flow rate independence.

Various researchers have done extensive theoretical and experimental studies analyzing the advantages of nanoelectrodes over microelectrodes. Edmund et.al. theoretically showed that hemispherical nanoelectrodes with radii less than $50 \mathrm{~nm}$ show reduced capacitance and a rapid potential drop from outer Helmholtz plane in diffusion domains ${ }^{[22]}$. The analysis at this level are expected to have enhanced driving forces (better mass transport) compared to electrodes larger than $100 \mathrm{~nm}$ which is useful for detection of extremely low concentrations of analytes. Amatore et. al. have conducted theoretical and experimental studies on the influence of natural convection due to thermal gradients and macroscopic vibrations on diffusional domains of microelectrodes ${ }^{[23]}$. The study finds that the natural convectional forces influence voltammetry signal at microelectrodes which are reputed to be immune and call for further decreasing the size of electrodes to nano domain to reduce the effects. There have been studies on optimizing nanoelectrode array geometric parameters which effect the electrochemical response by studying the qualitative influence of mass transport, effect of scan rate on the signal by changing the parameters such as nanoelectrode distribution and its radius ${ }^{[24-29]}$. Godino et. al. state that mass transport properties of a nanoelectrode heavily depend on its position in an array ${ }^{[30]}$. The inner electrodes in an array are completely surrounded by the neighboring electrodes which reduces the quantity of the analytes reaching them by diffusion compared to the electrodes at perimeter where the radial domains prevail for longer periods. Hence, the authors call for formation of small NEAs (leading to more edge effect) or an array with sufficiently large inter electrode spacing allowing for diffusional independence. Theoretical and experimental studies by Menshykau et. al shed light 
on diffusional independence at the nanoelectrode arrays and conclude that irrespective of the dimensions of electrode array the diffusional independence is valid only for a finite time scale ${ }^{[31]}$.

\subsection{Nanosphere Lithography}

Nanosphere lithography (NSL) is a fabrication technique utilized to make nanostructures with different materials and shapes at micro and nano scale. It is one of the most flexible, easy to use, inexpensive, high throughput and material general procedure to make well ordered 2 dimensional nanostructures. Standard lithographic techniques such optical lithography, electron beam lithography, ion beam lithography and x-ray lithography need expensive instrumentation, have low throughput, are not material general and are labor intensive. In one of the first papers, H.W. Deckman and J.H. Dunsmuir described new methods of microfabrication for making random and periodic metallic particles ${ }^{[32,33]}$. A monolayer of polystyrene spheres was formed using spin coating technique by optimizing the spin speeds and sphere concentrations. Since then NSL has been used extensively to produce periodic structures on a large area ${ }^{[34]}$. Different shapes such nanodots, nanotriangles, nanoholes, nanorings, 3D porous mesh of different materials on varied substrates have been fabricated by manipulating their sizes, spheres and inter particle spacing ${ }^{[35-40]}$. By etching the polystyrene monolayer using reactive ion etching, Haginoya et.al. published one of the first papers on fabricating nanohole array in silicon and creating a relationship between the polystyrene bead etching time and hole diameter ${ }^{[41]}$. Spin coating ${ }^{[42,43]}$ and dip

coating ${ }^{[44,45,46]}$ are two of the most common techniques to form the colloidal monolayer on a substrate. Both the techniques have ability for scaling to large scale fabrication and are easy to use for practical applications. However, a defect free and densely packed polystyrene monolayer has been formed with better controllability using dip coating. 
Dip coating is a technique based on the concept of Langmuir-Blodgett films (LB film) where a closely packed monolayer of colloids is formed by controlling the surface tension at the air-water surface $^{[47-49]}$. Molecules at the surface of the air-liquid interface experience imbalance forces and a net attractive force is acted upon them towards the liquid to minimize the surface area. This net effect produces a free surface energy and causes surface tension ${ }^{[50]}$. Polar liquids such as water have high surface tension due to intermolecular interactions. Changes in temperature or any presence of contamination on the surface of the liquid greatly lowers the surface energy. Amphiphilic molecules such as surfactants are added to lower the surface energy at the interface. When a surfactant is added, it accumulates at the air-liquid interface causing a reduction in the net surface free energy hence reducing the surface tension ${ }^{[51]}$. The LB film theory has been successfully applied to prepare densely packed monolayers of colloidal micro and nanosphere beads on water surface ${ }^{[52-55]}$. Thin film micropatterning using dip coating have been studied to fabricate periodical micro and nanostructures ${ }^{[46,56]}$.

1D, 2D and 3D nanostructures made from NSL have been extensively used for varied applications. Size varying nanotriangles for optical studies ${ }^{[57-58]}$, nanopillars with varied dimensions ${ }^{[59-62]}$, multi-layer structures using polystyrene as mask ${ }^{[63-65]}$, nanodots by thermal annealing of nanotriangles ${ }^{[66]}$, nanopyramid arrays ${ }^{[67]}$. Also, nanostructures have been fabricated for varied applications such as surface plasmon resonance, photovoltaics, photonic crystals, changing surface chemistry, optoelectronic devices, photocatalysis, etc. ${ }^{[68-73]}$. 


\subsection{Nanoelectrode Array as Chemical and Biological Sensors}

Nanoelectrode arrays fabricated using various techniques have been used as electrochemical sensors for chemical and biological sensing applications. Carbon based materials and noble metals have often been used for these applications because of their chemical inertness and biocompatibility. Noble metal based micro and nanoelectrodes have been used to detect toxic chemicals for real time environmental monitoring. Feeney et.al ${ }^{[74]}$ have used gold microelectrodes for real time detection of Arsenic, a toxic metal, in groundwater with the limit of detection $0.05 \mathrm{ppb}$ and large linear response. The electrodes have been fabricated using standard photolithography. Highly toxic mercury $\left(\mathrm{Hg}^{2+}\right)$ has been detected with linear response on $10 \mathrm{ppb}$ to $200 \mathrm{ppb}$ with a LOD of $3.2 \mathrm{ppb}$ with real time analysis on gold microelectrodes with $5 \mu \mathrm{m}$ diameter ${ }^{[75]}$. Another toxic metal chromium ${ }^{[76]}$ has been detected using gold microelectrodes with radius of $10 \mu \mathrm{m}$ and inter electrode spacing of $100 \mu \mathrm{m}$ having linear range of 13-428 $\mu \mathrm{M}$ with a LOD of $3.4 \mu \mathrm{M}$.

Recently, electrochemical based biosensors have begun to make transition from the microelectrodes to nanoelectrodes. An array of carbon nanotubes fabricated using e-beam lithography have been used for DNA and RNA detection showing dramatic improvement in sensitivity by reducing the electrode density ${ }^{[77-78]}$. Gold nanowell array fabricated by e-beam lithography for high sensitivity detection of DNA were conducted by Lee et. al. ${ }^{[79]}$. A $5 x 5$ array of 60 to $120 \mathrm{~nm}$ radii gold nanoelectrodes was integrated in chip based microfluidics platform for prostate-specific antigen detection ${ }^{[80]}$. The electrodes were made using focused ion beam lithography and a detection limit of $10 \mathrm{pg} / \mathrm{ml}(\sim 270 \mathrm{fM})$ was obtained which is approximately 30000 copies of prostate specific antigen. Ogorevc et. al. reported the use of carbon fiber nanotubes (CFNTs) to detect dopamine and the calibration plots obtained were linear over the range from 0.5 
$\mu \mathrm{M}$ to $0.1 \mathrm{mM}$, with a LOD of $0.1 \mu \mathrm{M}^{[81]}$. Clearly, nothing much has been done with biological detection using gold based nanoelectrode array.

\subsection{Gaps in the Literature}

Based on the literature review, though there have been extensive theoretical studies on electrochemistry at nanoelectrodes nothing much experimental work has been conducted to produce a large area nanoelectrode. This is due to the fact of lacking options to fabricate nanoelectrodes on a large scale with ease and high reliability. Due to lack of an easy and reliable process to fabricate small dimension electrode, in this work, a new procedure to fabricate a large area nanoelectrode array will be presented to overcome the problems mentioned above. Electrochemistry of NEAs will be investigated to understand the changes in the signal with changing geometric factors of electrodes. The results obtained will provide guidelines for a better electrochemical sensor for biological applications. The best possible nanoelectrode array design will be used for detection of dopamine in presence of ascorbic acid as an application. 


\section{Fabrication and Electrochemical Testing of Nanoelectrode Array using Nanosphere Lithography}

\subsection{Introduction}

As mentioned previously, there are a very few robust and reliable approaches to fabricate a large scale array of nanoelectrodes. In this chapter, a new procedure to fabricate nanoelectrodes using nanosphere lithography is discussed. A systematic alteration of electrode diameter and the spacing between the electrodes is made to record the changes in electrochemical signal. In the following sections, an overview on experimental design, materials and instruments used, fabrication procedure of NEAs, electrochemical experiments and analysis for nanoelectrode arrays is discussed.

\subsection{Experimental Design}

In this study, gold nanoelectrodes with $\mathrm{SiO}_{2}$ as an inter electrode filling for insulation are fabricated. To make the study simple, the nanoelectrodes are divided into two categories. Category 1 has nanoelectrode array with a constant electrode size (d) and varying inter electrode spacing (x). Category 2 has nanoelectrode array with a constant inter electrode spacing (x) and varying

electrode size (d). As illustrated in Figure 3.1, nanoelectrodes in category 1 have a constant electrode size (d) of $100 \mathrm{~nm}$ and varied inter electrode spacing of $200 \mathrm{~nm}, 500 \mathrm{~nm}$ and $1000 \mathrm{~nm}$. In category 2, the inter electrode spacing of electrodes is kept constant at $1000 \mathrm{~nm}$ and electrode size is varied as $100 \mathrm{~nm}, 400 \mathrm{~nm}$ and $700 \mathrm{~nm}$. The dimensions of the nanoelectrodes are determined with fabrications limitations under consideration. 

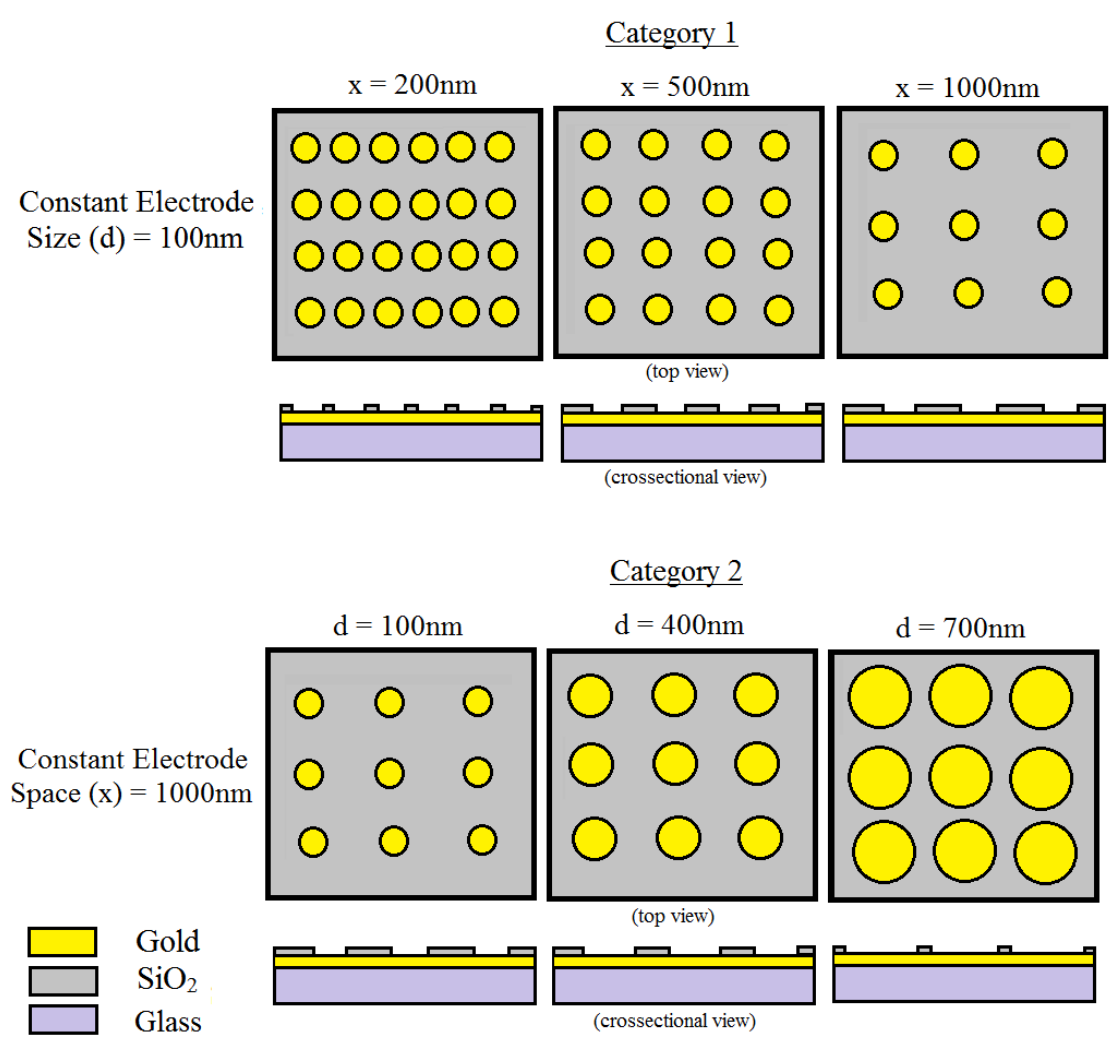

Figure 3.1: 2 Categories of fabricated nanoelectrodes.

The size and spacing between the electrodes greatly affect the overlapping abilities of the diffusional domains at the electrode surface. With increasing distance between the electrodes, the radial diffusional domains become more independent leading to a steady transportation of analytes to the electrode surface. From the simulation studies, shown in Figure 3.2, steady state currents are obtained with farther electrodes. Bulk electrodes produce peak shaped curves, a characteristic trait of linear diffusion, due to limited transport of analytes to the electrode surface [67]. Further in this chapter, electrodes are prepared to study the phenomenon of diffusional independence and its effects on electrochemical signal. 


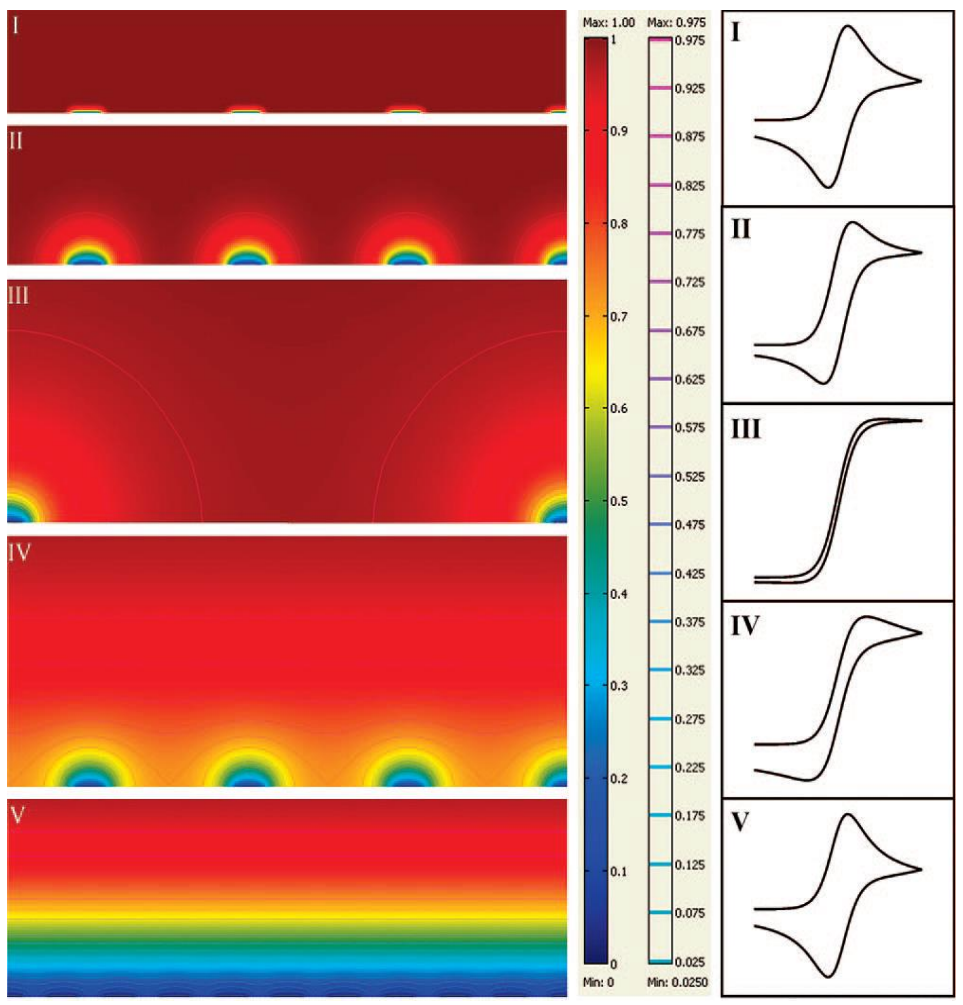

Figure 3.2: Simulations studies showing diffusional domain independence due to increasing electrode spacing ${ }^{[27]}$.

\subsection{Experimental Section}

\subsubsection{Materials}

Following materials have been used for the fabrication of nanoelectrodes: plain microscope glass slides (Fischer Scientific Inc., USA), gold and titanium pellets a metal source for electron beam evaporation (Kurt J. Lesker Company, USA), XR-1541 ebeam resist in MIBK (Dow Corning, USA), 5000 series polystyrene beads with a diameter of $200 \mathrm{~nm}, 500 \mathrm{~nm}$ and $1000 \mathrm{~nm}$ (Thermo Scientific Inc., USA). 
Following materials have been used for electrochemical testing: potassium hexacyanoferrate (II) trihydrate $<98.5 \%$ (Sigma Aldrich, USA), potassium hexacyanoferrate (III) 99\% (Sigma Aldrich, USA), phosphate buffer saline tablets (Sigma Aldrich, USA) and deionized water produced by the Milli-Q Integral 3/5/10/15 system (18.2 M $\Omega \cdot \mathrm{cm}$, Millipore Corp., USA).

\subsubsection{Instruments}

Following instruments have been used for fabrication of nanoelectrodes: Pro Line PVD 200 electron beam evaporator for gold and titanium deposition on glass slides (Kurt J. Lesker Inc., USA), Model 400 spin coater for spinning XR-1541 (Laurell Technologies Inc., USA), March PX250 oxygen plasma asher for etching polystyrene spheres (Nordson March Inc., USA) and Minilock III RIE - ICP for etching the insulating $\mathrm{SiO}_{2}$ layer (Trion Technology Inc, USA).

Following instruments have been used to characterize the nanoelectrodes: JSM-7600F field emission scanning electron microscope for taking high resolution images (JEOL USA Inc., USA), M-2000U ellipsometer (J.A. Woolam Co. Inc., USA) to find the thickness of $\mathrm{SiO}_{2}$ insulation layer, PHI 5000 VersaProbe X-ray photon spectroscope (Physical Electronics Inc., USA) to find the quality of the insulator $\left(\mathrm{SiO}_{2}\right)$ layer.

Following instruments have been used for electrochemical testing: Gamry reference 600 potentiostat and Gamry echem framework software (Gamry Instruments Inc., USA) for signal recording, BASi C3 cell stand (BASi Inc., USA) for electrochemical analysis. 


\subsubsection{Fabrication of Nanoelectrodes}

An important aspect to get a large area, periodically ordered array of electrodes is to obtain a closely packed, defect free, evenly ordered monolayer of polystyrene spheres on the surface of the substrate. In this study, dip coating method is opted to form a compact monolayer of polystyrene spheres based on a previous study by Li et. al. ${ }^{[46]}$. Taking the advantage of flexibility with nanosphere lithography, a multi-step top-down fabrication approach is developed using standard semiconductor microfabrication processes. A step by step fabrication procedure of a gold nanoelectrode array, as illustrated in Figure 3.3, using nanosphere lithography as follows:

a) Substrate Cleaning: A microscopic glass slide is cut into $1 \mathrm{~cm} \times 1 \mathrm{~cm}$ pieces and ultrasonicated in acetone, methanol and DI water sequentially for 5 min each. Then the glass pieces are blown dried using ultra-pure nitrogen gas and heated at $180^{\circ} \mathrm{C}$ for 15 min to remove any moisture from the surface.

b) Metal Deposition: Once the samples are cooled down to the room temperature, $10 \mathrm{~nm}$ of titanium (Ti) and $100 \mathrm{~nm}$ of gold ( $\mathrm{Au}$ ) are deposited using electron beam evaporation physical deposition under high vacuum $\left(10^{-7}\right.$ torrs $)$. Deposition of the gold metal in the electron beam evaporation is highly susceptible to a phenomenon called 'spitting' where droplets of melted gold 'spit' from the source material. Hence, the gold source is evenly and completely melted with slow ramping currents before deposition to avoid any spitting. Titanium layer acts as an adhesion layer between the gold layer and glass slide. 
(a)

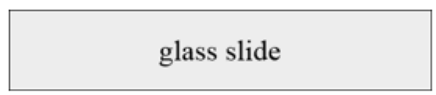

(b)

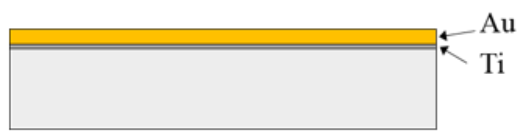

(c)

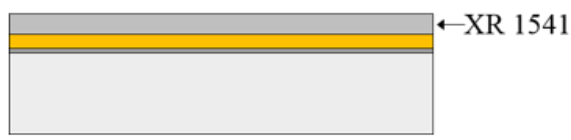

(d)

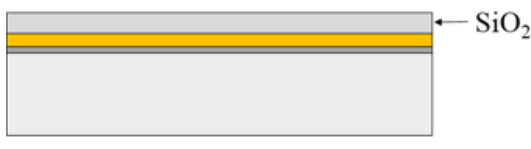

3S5 3155

(e)

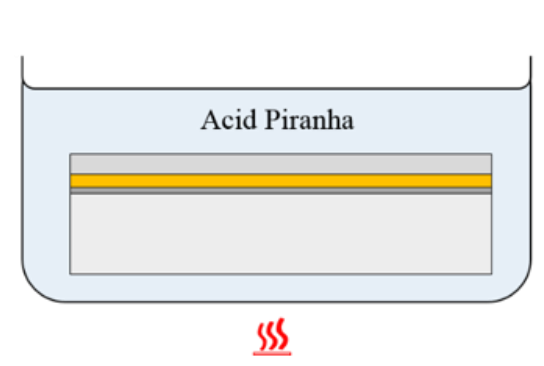

(f)

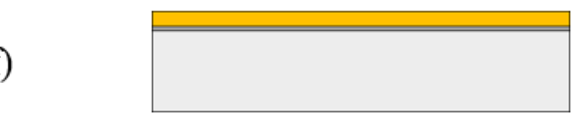

g)

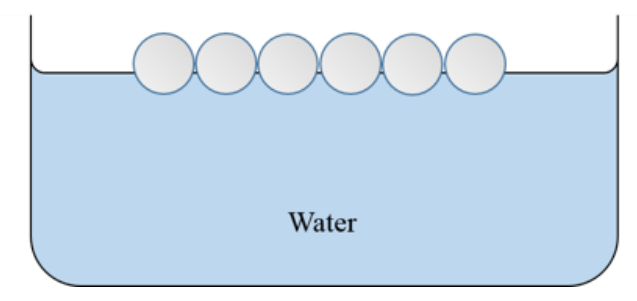

(h)

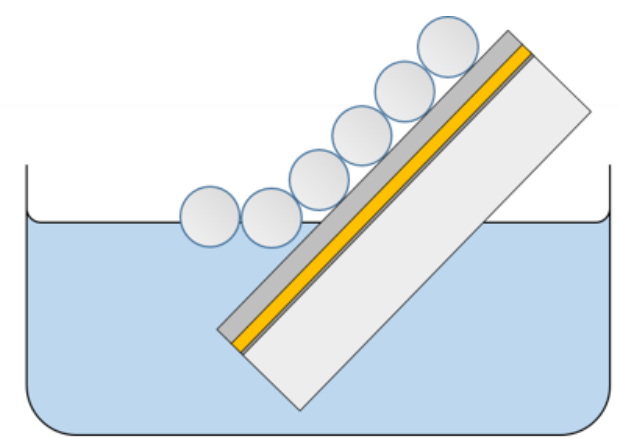

(i)

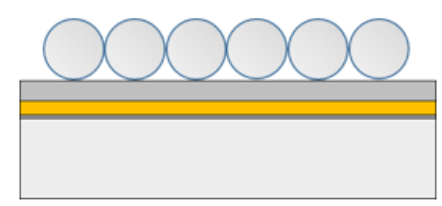

(j)

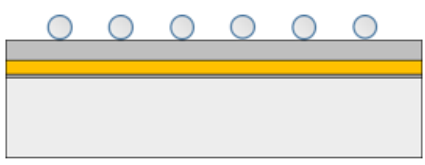

(k)

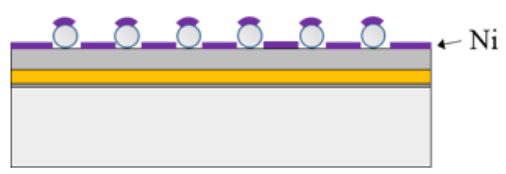

(1)

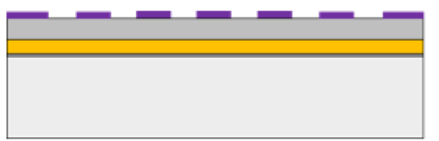

(m)
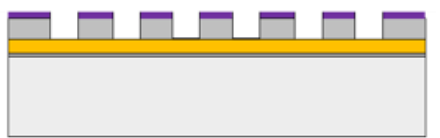

(n)

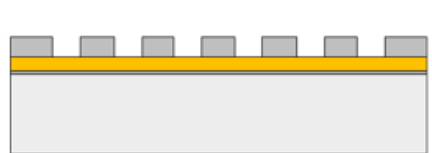

Figure 3.3: NEA fabrication procedure.

(All the dimensions in the figure are not relative. Certain parts have been magnified for better visibility.) 
c) XR-1541 Spin Coating: After the metal deposition, spin on dielectric XR-1541 is spin coated on to the substrate. XR-1541 is a resist used for e-beam lithography consisting of hydrogen silsesquioxane (HSQ) diluted in the methyl isobutyl ketone (MIBK) as solvent. The XR-1541 is spin coated over the substrate at $4000 \mathrm{rpm}$ for $1 \mathrm{~min}$ and heated at $95^{\circ} \mathrm{C}$ for 4 min to remove excess solvent.

d) $\underline{\mathrm{SiO}}_{2}$ Formation: After coating the substrate with $\mathrm{XR}-1541$, it is annealed at $400^{\circ} \mathrm{C}$ for 1 hour under nitrogen gas as an inert environment. At such high temperatures, the HSQ in the resist is decomposed to form silicon dioxide $\left(\mathrm{SiO}_{2}\right)$. The formation of $\mathrm{SiO}_{2}$ is verified by XPS analysis. The thickness of the layer is found to be approximately $100 \mathrm{~nm}$ using ellipsometry.

e) Hydrophilic Treatment: After formation of the insulation layer, the substrates are placed in a 7:3 ratio mixture of sulfuric acid $\left(\mathrm{H}_{2} \mathrm{SO}_{4}\right)$ and hydrogen peroxide $\left(\mathrm{H}_{2} \mathrm{O}_{2}\right)$ (also called as 'Acid Piranha') at $80^{\circ} \mathrm{C}$ for 1 hour. (Caution: use acid piranha solution in fume hood only. It is highly reactive, extremely corrosive and is to be handled with extreme caution). Due to the attachment of $-\mathrm{OH}$ bonds, the surface of the electrode $\left(\mathrm{SiO}_{2}\right.$ layer $)$ becomes extremely hydrophilic.

f) Cleaning and Storage: After piranha treatment, the substrates are thoroughly rinsed with DI water to remove any residuals from the acid treatment. After cleaning, the samples are stored under DI water until the next step to keep the hydrophilicity intact.

g) Monolayer Formation: Now, the polystyrene (PS) beads as received are added with ethanol in the ratio 3:2, 1:1 and 2:3 (PS:ethanol) with the PS bead diameter $200 \mathrm{~nm}, 500 \mathrm{~nm}$ and $1000 \mathrm{~nm}$ respectively. The solution is mixed well and is dropped onto a hydrophilic 
$2 \mathrm{~cm} \times 2 \mathrm{~cm}$ silicon substrate and let it spread evenly. The silicon substrate is made hydrophilic using the procedure in step (e). As soon as the PS bead solution is evenly spread, the silicon substrate is carefully and slowly dipped into a shallow petri dish filled with DI water. The water is added with 4 drops of $1 \mathrm{mM}$ sodium dodecyl sulfate as a surfactant which is used to reduce the surface energy of the water. Once the PS bead solution is transferred into the petri dish, the water is allowed to settle and eventually a monolayer of PS beads is formed on the surface of the DI water. This process is based on the Langmuir-Blodgett film theory as discussed in the chapter 2.

h) Transfer of Monolayer onto the Substrate: After the formation of the monolayer, the hydrophilic $\mathrm{SiO}_{2}$ coated gold substrates are carefully dipped into the water without creating much turbulence. The monolayer on the water is carefully transferred onto the substrate by pulling the substrate carefully from underneath the floating monolayer. Caution should be taken while transferring as pulling the substrate too fast will lead to breaking of the monolayer.

i) Drying and Formation of a Compact Monolayer: Once the monolayer is transferred, the substrates are allowed to dry at a slightly inclined angle with a lid placed over them. At a tilted angle, evaporation of the water between the PS beads leads to a capillary action and electrostatic attraction helping in formation of a closely packed monolayer of PS beads ${ }^{[82]}$. The substrates are allowed to dry for $4-5$ hours at about $60^{\circ} \mathrm{C}$ in a convection oven (Figure 3.4). 

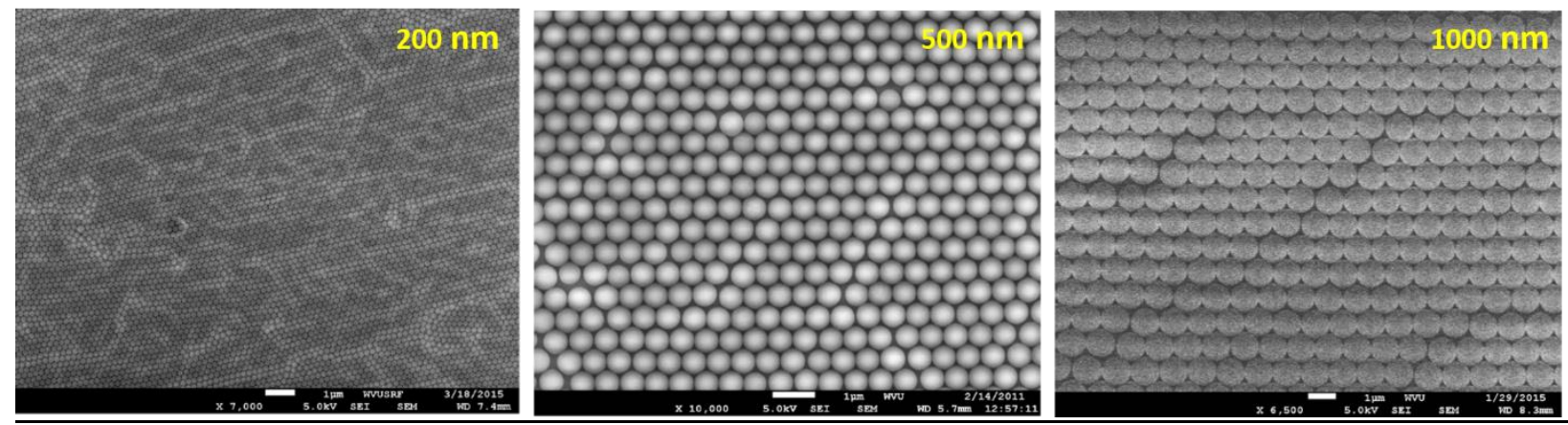

Figure 3.4: Closely packed monolayer of PS beads of diameter $200 \mathrm{~nm}, 500 \mathrm{~nm}$ and $1000 \mathrm{~nm}$.

j) Polystyrene Etching: The polystyrene monolayer on the substrate are carefully etched in an oxygen plasma asher to shrink the size of the microspheres to the desired dimensions. Oxygen ions in the plasma react with the polystyrene and the product is removed away from the surface. The parameters used for the oxygen plasma ashing are $30 \mathrm{~mW}$ (power), 110 mTorr (plasma gas pressure) and etching time is calculated depending on the dimensions required (Figure 3.5). 

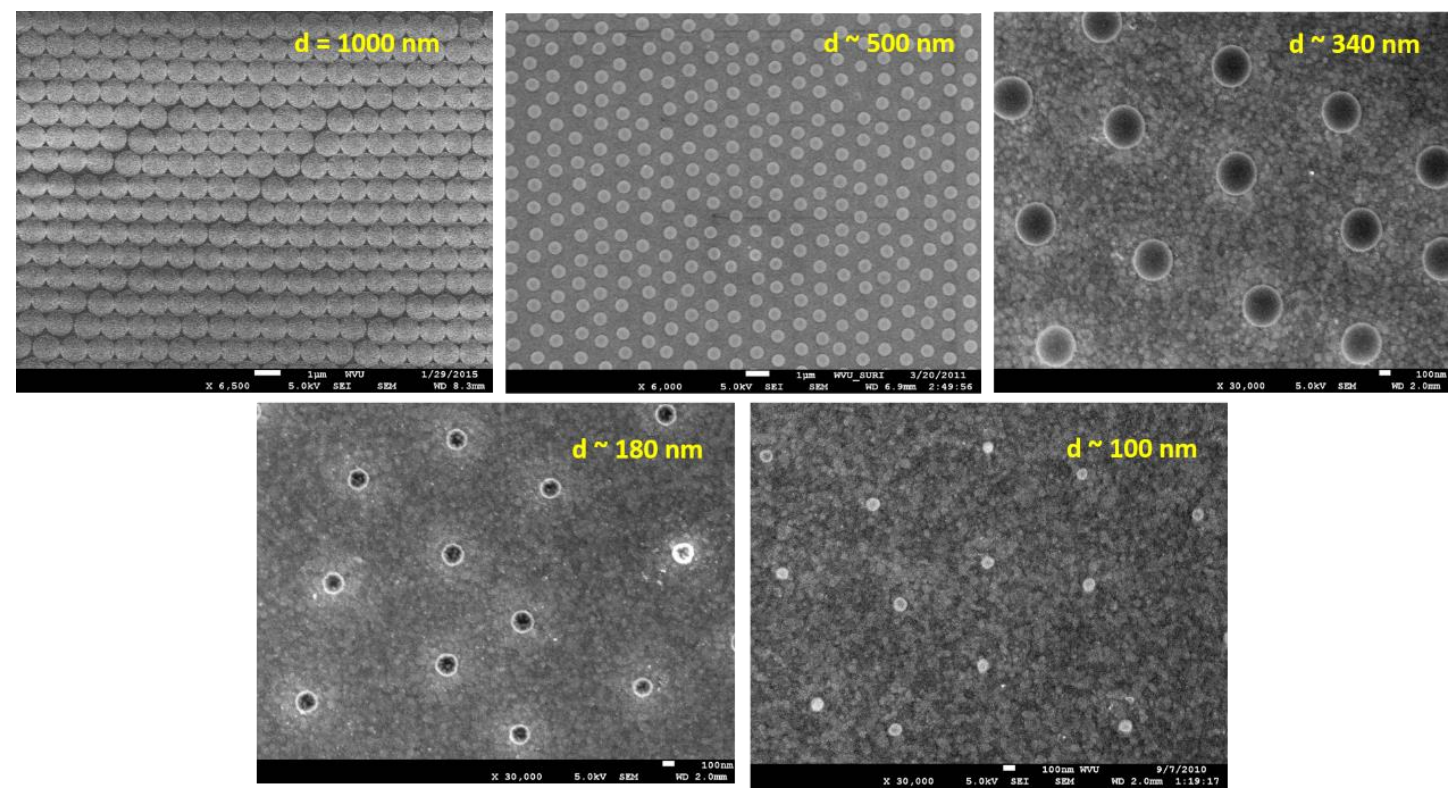

Figure: 3.5: Size reduction of a $1000 \mathrm{~nm}$ diameter PS beads monolayer in an oxygen plasma asher.

k) Nickel Etch Mask: Using etched polystyrene beads as a shadow mask, $15 \mathrm{~nm}$ of nickel metal (Ni) is deposited using electron beam evaporation.

1) Removal of Beads: After deposition of $\mathrm{Ni}$, the substrate is gently sonicated for 2-3 minutes in ethanol to remove all the polystyrene beads from surface to remain with a periodic $\mathrm{Ni}$ hole array pattern with the hole diameter equal to the diameter of the removed polystyrene.

m) Etching of $\mathrm{SiO}_{2}$ Layer: $\mathrm{The}^{\mathrm{SiO}_{2}}$ insulating layer is selectively etched using inductively coupled plasma reactive ion etching (ICP-RIE) with $\mathrm{Ni}$ as mask. The exposed $\mathrm{SiO}_{2}$ layer at the holes in Ni mask is etched isotropically and the etching times are calibrated until the underlying gold layer is exposed. The diameter of the exposed gold electrode is approximately equal to diameter of the Ni hole.

n) Removal of Ni mask: Once the underlying gold electrodes are exposed, the Ni mask on the top of the insulating layer is removed by wet etching process. The substrates are dipped in 
a mixture of DI water, nitric acid $\left(\mathrm{HNO}_{3}\right)$, acetic acid $\left(\mathrm{CH}_{3} \mathrm{COOH}\right)$ and sulfuric acid $\left(\mathrm{H}_{2} \mathrm{SO}_{4}\right)$ in the proportions 10:5:5:2 ${ }^{[83]}$. The samples are stirred for 1-2 min or until the color of metallic nickel is visibly gone. Later the samples are thoroughly washed in DI water to remove any remains of the etchant and blown dry with nitrogen before further usage.

As mentioned above, spin on dielectric XR-1541 was used as a precursor to form $\mathrm{SiO}_{2}$ insulating layer. It is an easy method to form a very flat post annealing insulating surface with an excellent profile coverage. $\mathrm{SiO}_{2}$ layer deposited using electron beam evaporation and plasma enhanced chemical vapor deposition (PECVD) have proven to have fine porosity with producing redox signals in electrochemical testing. As shown in the Figure 3.6, XPS scans show the peaks of an annealed XR-1541 sample exactly match with a $\mathrm{SiO}_{2}$ spectra., The spectrum has been adjusted to carbon peaks at $284.4 \mathrm{eV}$. 

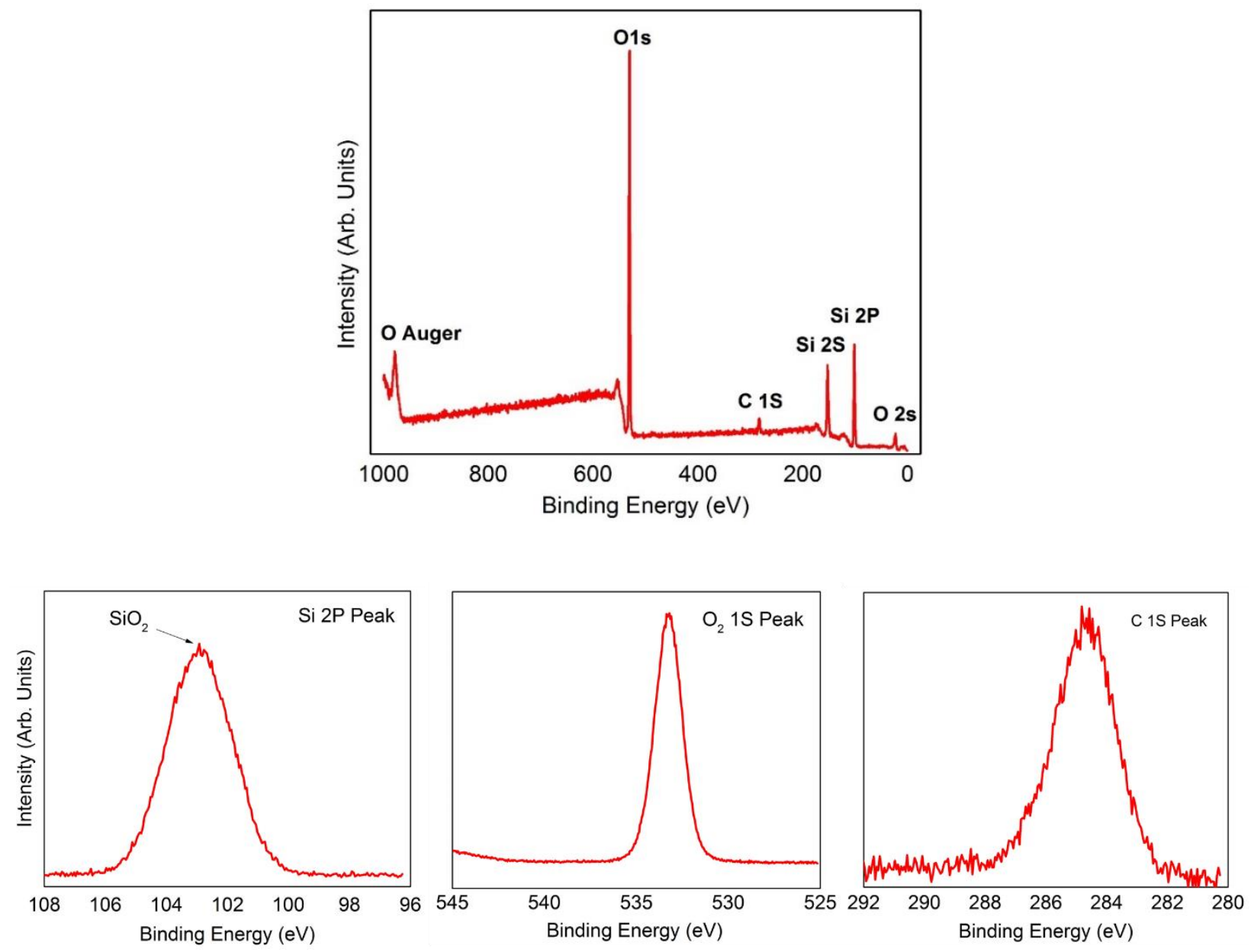

Figure 3.6: XPS survey scan and elemental scans of annealed XR-1541 layer.

XR-1541 coated silicon samples were spun at $4000 \mathrm{rpm}, 5000 \mathrm{rpm}$ and $6000 \mathrm{rpm}$ and annealed to find a spin speed vs. post annealed thickness plot, shown in Figure 3.7 (a). All the $\mathrm{SiO}_{2}$ layer thickness measurements were conducted using an ellipsometer with a Cauchy fitting with urbach absorption. Figure 3.7 (b) shows an experimental and fitted ellipsometer plot for a $101.6 \mathrm{~nm}$ thick $\mathrm{SiO}_{2}$ layer. Not much variation in thickness of $\mathrm{SiO}_{2}$ is observed with increasing XR-1541 spin coating speeds from $4000 \mathrm{rpm}$ to $6000 \mathrm{rpm}$. 


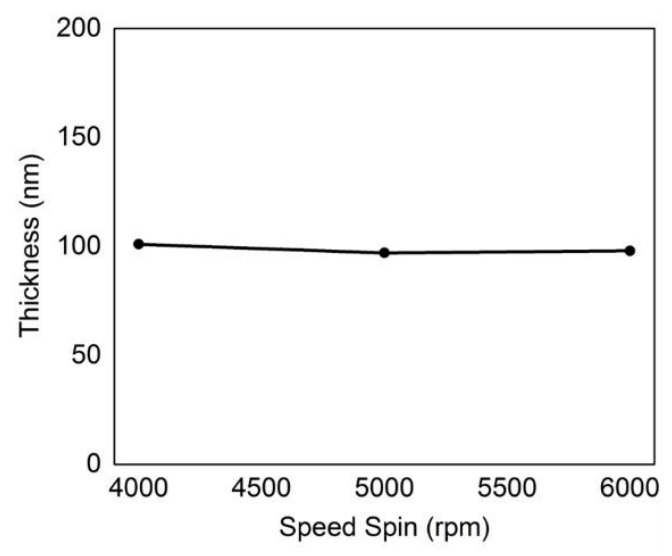

(a)

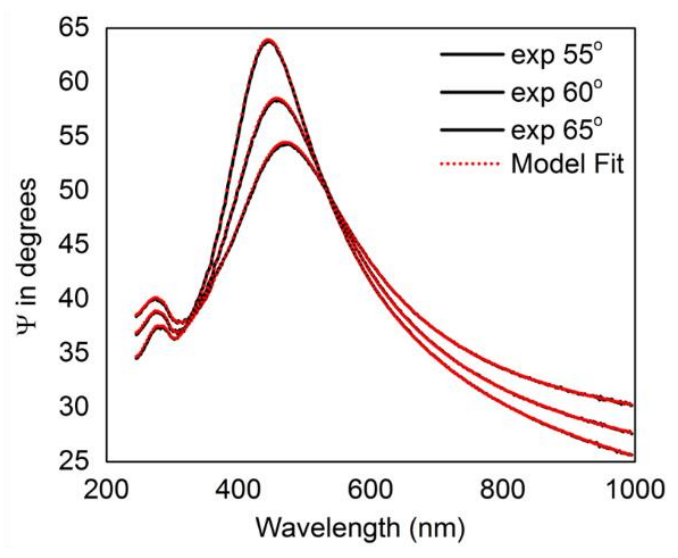

(b)

Figure 3.7: (a) XR-1541 spin speed vs. $\mathrm{SiO}_{2}$ layer thickness and (b) ellipsometry curves for $\mathrm{SiO}_{2}$ over $\mathrm{Si}$ with Cauchy fitting.

A mixture of tetrafluoromethane $\left(\mathrm{CF}_{4}\right)$ and oxygen $\left(\mathrm{O}_{2}\right)$ in the ratio 9:1 (18 $\left.\mathrm{sscm}: 2 \mathrm{sscm}\right)$ respectively has been used to find the selective etching rates of nickel and silicon dioxide in ICP-RIE. Multiple $\mathrm{Si}$ substrates coated with $500 \mathrm{~nm}$ thick $\mathrm{SiO}_{2}$ layer (formed by annealing multiple layers of XR-1541) and $100 \mathrm{~nm}$ thick Ni were used as test samples to find the etch rates. An ICP power of $400 \mathrm{~W}$ and RIE power of $100 \mathrm{~W}$ was used as etching parameters and a stylus profilometer was used to find the etch depths. The calculated etching rates of $\mathrm{SiO}_{2}$ and $\mathrm{Ni}$ are shown in the Figure 3.8. Each point on the plot is an average of the data obtained from 5 separate etch processes taken at different times. Due to its extremely low etching rates, it is very evident from the data that $\mathrm{Ni}$ acts as an excellent mask to etch $\mathrm{SiO}_{2}$. The time used to etch $100 \mathrm{~nm}$ of $\mathrm{SiO}_{2}$ was about $23 \mathrm{sec}$, a little more than the time obtained from the plot to make sure the pores are well opened. Figure 3.9 shows a nanoelectrode array after removal of Ni mask, with approx. $100 \mathrm{~nm}$ diameter holes in the $\mathrm{SiO}_{2}$ layer with revealing gold surface beneath. 


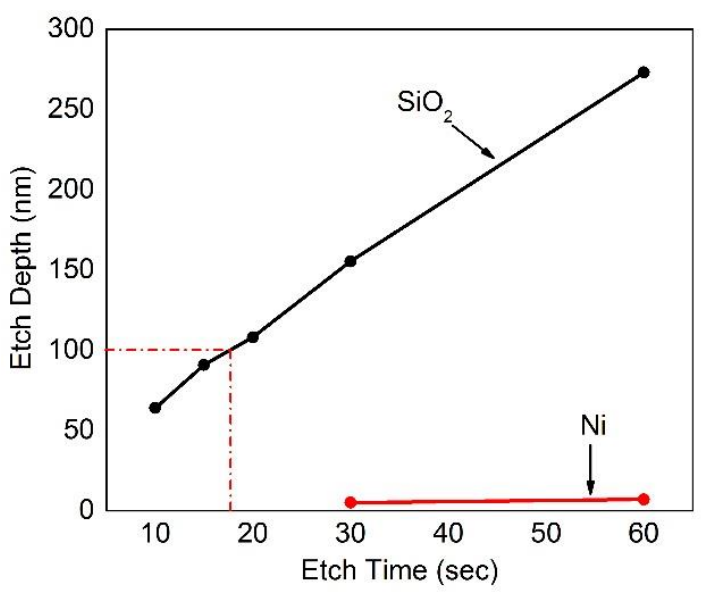

Figure 3.8: Etching rates of $\mathrm{SiO}_{2}$ and $\mathrm{Ni}$ in ICP-RIE.

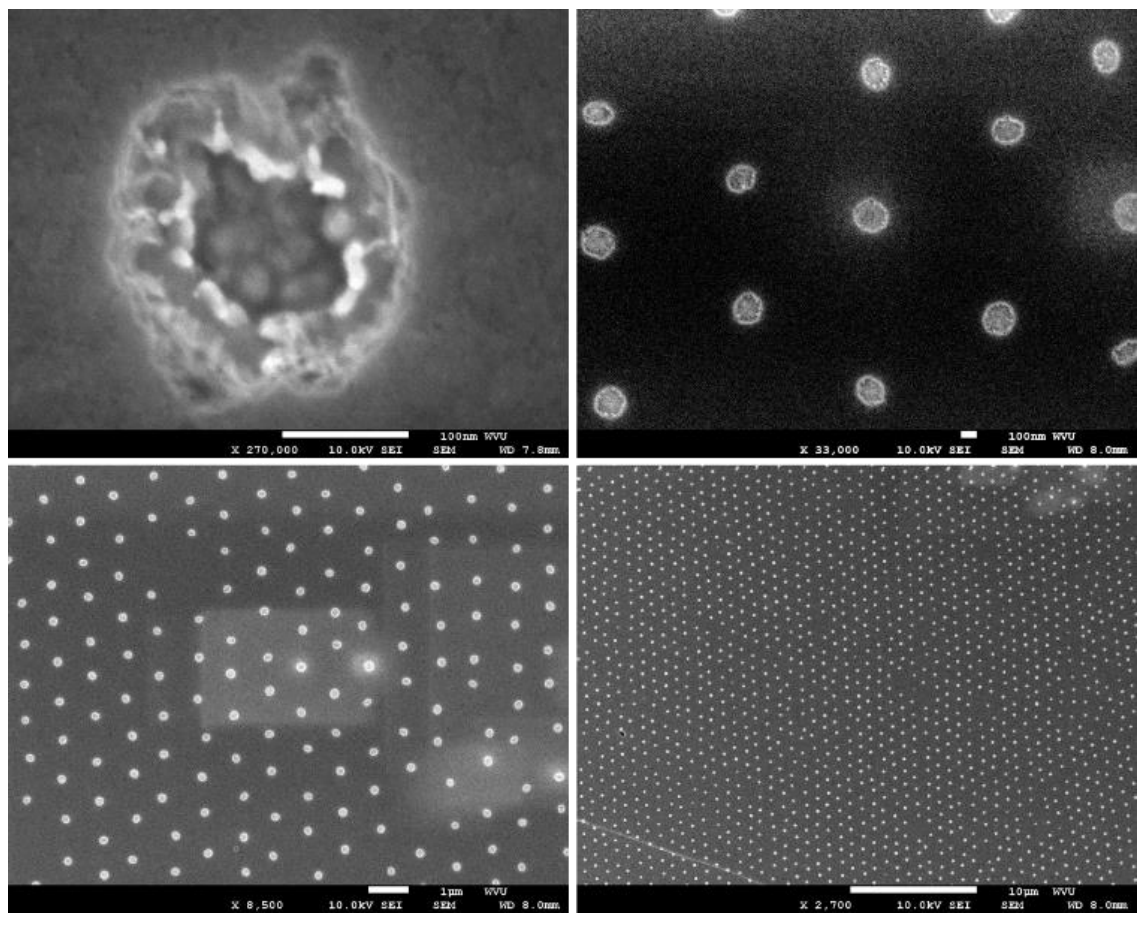

Figure 3.9: A nanoelectrode array of $d=100 \mathrm{~nm}$ and $\mathrm{x}=1000 \mathrm{~nm}$. 
As mentioned earlier, two categories of nanoelectrode arrays were prepared using the fabrication procedure explained above. Figure 3.10 shows the SEM images of category 1 electrode set which has a constant electrode diameter (d) of $100 \mathrm{~nm}$ and a varying inter electrode spacing (x) of 200 $\mathrm{nm}, 500 \mathrm{~nm}$ and $1000 \mathrm{~nm}$. Figure 3.11 shows the SEM images of category 2 electrode set which has a constant inter electrode spacing (x) of $1000 \mathrm{~nm}$ and varying electrode diameter (d) of 100 $\mathrm{nm}, 400 \mathrm{~nm}$ and $700 \mathrm{~nm}$.
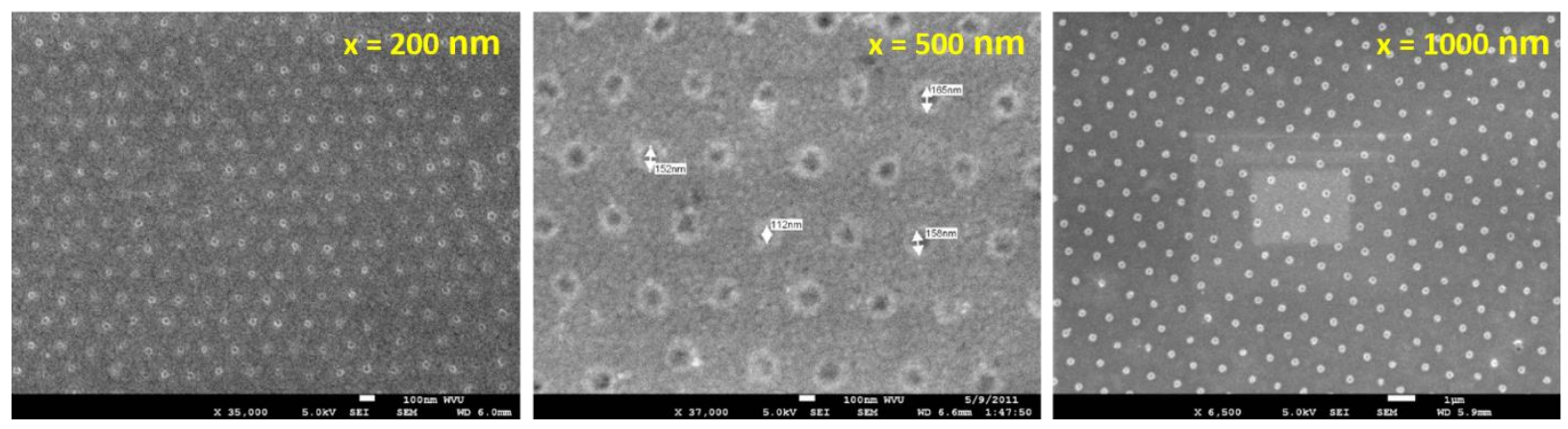

Figure 3.10: SEM images of Category 1 NEAs with a constant electrode diameter (d) of $100 \mathrm{~nm}$ and varying inter electrode spacing (x) of $200 \mathrm{~nm}, 500 \mathrm{~nm}$ and $1000 \mathrm{~nm}$.
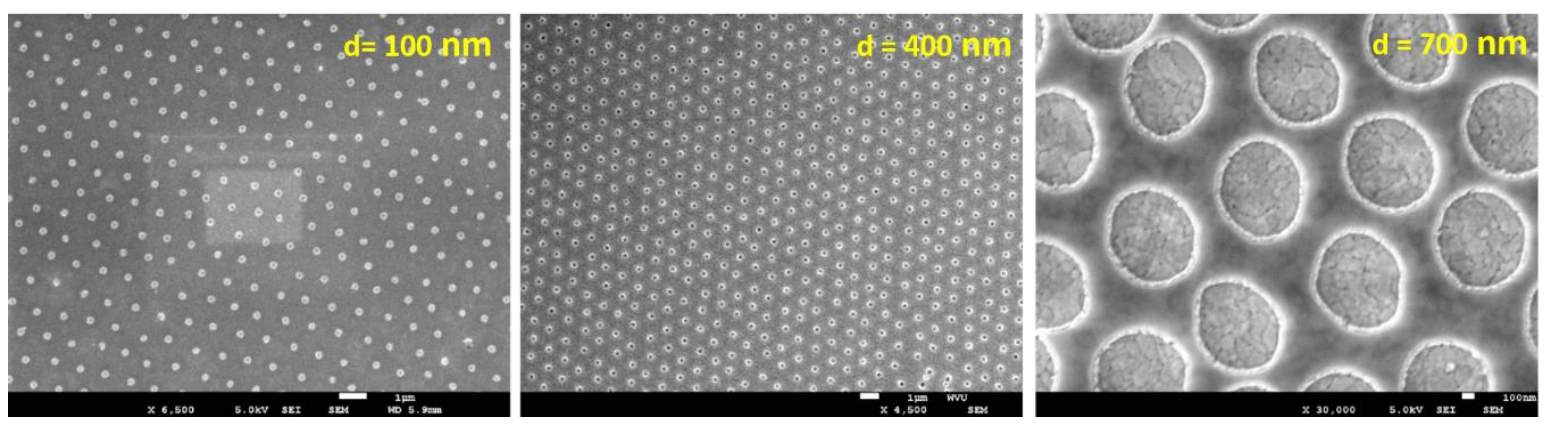

Figure 3.11: SEM images of Category 2 NEAs with a constant inter electrode spacing (x) of $1000 \mathrm{~nm}$ and varying electrode diameter (d) of $100 \mathrm{~nm}, 400 \mathrm{~nm}$ and $700 \mathrm{~nm}$. 


\subsubsection{Electrochemical Testing of Nanoelectrode Arrays}

Before any electrochemical testing was conducted, edges of all the electrodes (planar gold macroelectrode and nanoelectrode array substrates) were sealed with a Kapton tape (thick polyimide film with glue on one side) as shown in the Figure 3.12 (a). All the electrodes were sealed such that the center had a dimension of $1.5 \times 1.5 \mathrm{~mm}^{2}$ and edges were completely sealed on all sides except on the top. This serves two purposes. First is that every electrode has a consistent surface area exposed to the electrolyte. And second is that the sealing does not let the exposed gold layer at the edges, esp. for NEAs (Figure 3.13 (b)), to contribute to electrochemical signal in the experiments.

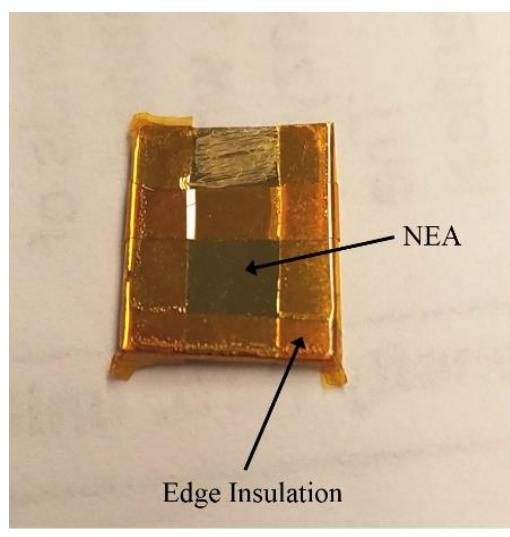

(a)

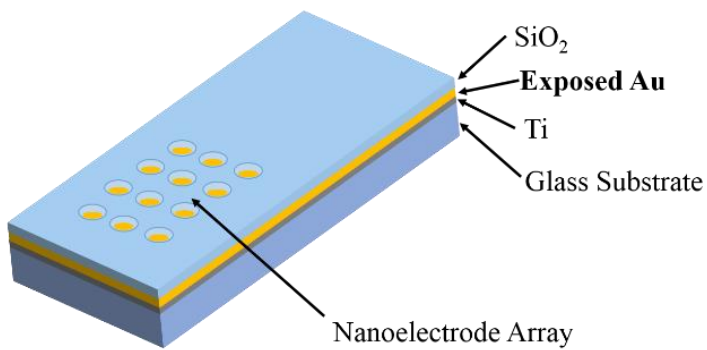

(b)

Figure 3.12: (a) Edge insulation for electrodes and (b) exposed gold layer at the electrode edges.

An image of the cell set up for all the electrochemical experiments is shown in the Figure 3.13. In all the experiments, a platinum $(\mathrm{Pt})$ mesh was used as a counter electrode and a silver/silver chloride $(\mathrm{Ag} / \mathrm{AgCl})$ electrode was used as a reference electrode. Platinum mesh electrode was 
cleaned with $0.1 \mathrm{M}$ nitric acid before every experiment and $\mathrm{Ag} / \mathrm{AgCl}$ reference electrode was always stored in $3 \mathrm{M}$ potassium chloride $(\mathrm{KCl})$ solution. The electrolyte used for the electrochemical analysis of the electrodes was a mixture of $1 \mathrm{mM}$ potassium ferricyanide $\left(\mathrm{K}_{3}\left[\mathrm{Fe}(\mathrm{CN})_{6}\right]\right)$ and $1 \mathrm{mM}$ potassium ferrocyanide $\left(\mathrm{K}_{4}\left[\mathrm{Fe}(\mathrm{CN})_{6}\right]\right)$ in phosphate buffer saline (PBS) solution. $\mathrm{Fe}^{2+}$ and $\mathrm{Fe}^{3+}$ ions act as electroactive species and PBS acts as the inert salt to reduce the migration effects and increasing the conductivity of the electrolyte. PBS solution matches the osmolarity and ionic concentrations of the human body and is a mixture of $10 \mathrm{mM}$ phosphate buffer, $2.7 \mathrm{mM}$ potassium chloride and $137 \mathrm{mM}$ of sodium chloride with $\mathrm{pH}$ 7.4. $\mathrm{Fe}^{3+}$ and $\mathrm{Fe}^{2+}$ ions (also called $\mathrm{F}^{3+} / \mathrm{Fe}^{2+}$ system) act as the redox couple for the cyclic voltammetry analysis described by the equation:

$$
\mathrm{Fe}^{3+}+\mathrm{e}^{-} \Leftrightarrow \mathrm{Fe}^{2+}
$$

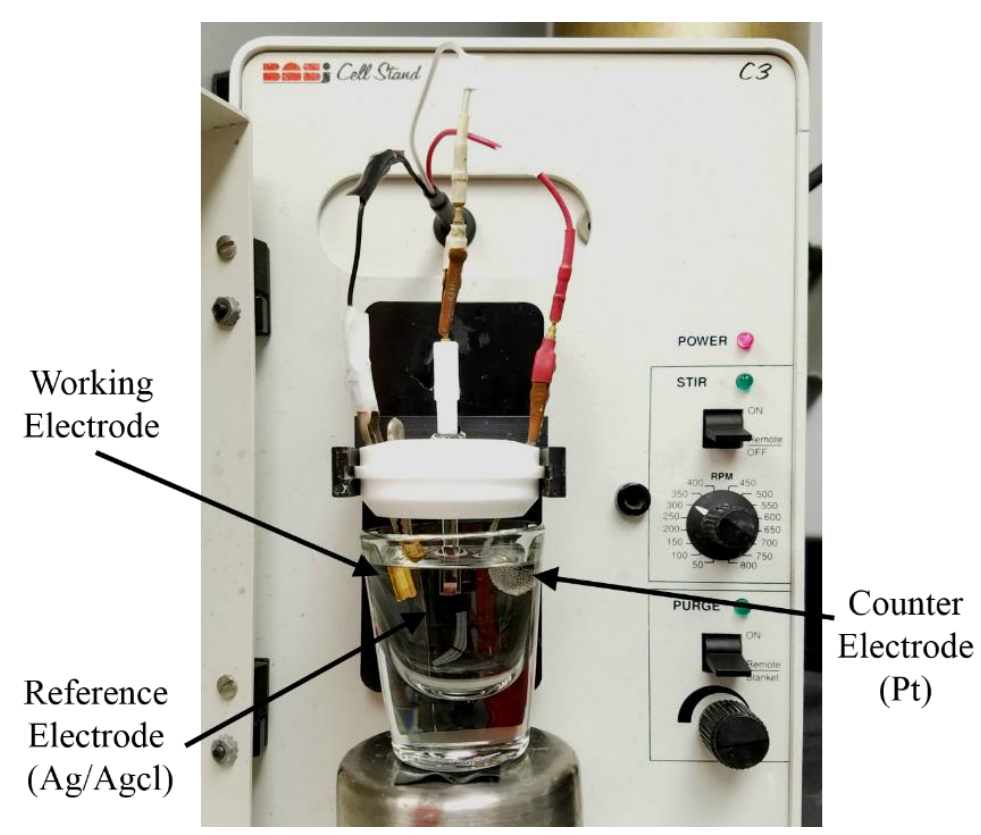

Figure 3.13: Cell set up for the electrochemical analysis. 
One of the important aspects of the testing is to make sure that there is no seepage of the electrolyte solution through the $\mathrm{SiO}_{2}$ insulation layer. Checking the quality of the $\mathrm{SiO}_{2}$ layer is very important as any tiny crack or incomplete coverage over gold electrode will generate a redox signal and defeat the purpose of the insulation layer. Several gold electrodes completely covered with the $\mathrm{SiO}_{2}$ layer with edge insulation were tested to check for any electrochemical signal. None of the tested electrodes produced any redox signal from the electrolyte as seen in the Figure 3.14 (a). Lack of any signal from the electrodes in the electrochemical redox window proves that the $\mathrm{SiO}_{2}$ insulation layer has no openings or leakages and has an excellent coverage over the gold surface.

Another important part of the testing of the electrodes is to check the quality of the electrode surface for both planar gold electrodes and nanoelectrode array electrodes. As electrochemistry is a surface analysis, any contamination or changes in surface chemistry will adversely affect the signal produced. Hence, while fabricating the electrode, extra care is taken to clean the electrode surface by thoroughly cleaning with DI water or using oxygen plasma to remove any organic residual. Electrochemical activation has proven to be a very effective in cleaning the electrode surface ${ }^{[84]}$. Using $0.5 \mathrm{M}$ of sulfuric acid as the electrolyte, a cyclic voltammetry scan was cycled between $0 \mathrm{~V}$ to $+1.5 \mathrm{~V}$ for 15 cycles. During the process, the gold at the surface is oxidized and reduced multiple times and is cleaned by producing a characteristic redox signal with a sharp reduction peak. Figure 3.14 (b) shows one such activation cycle for both planar bare gold macroelectrode and nanoelectrode array using sulfuric acid. 


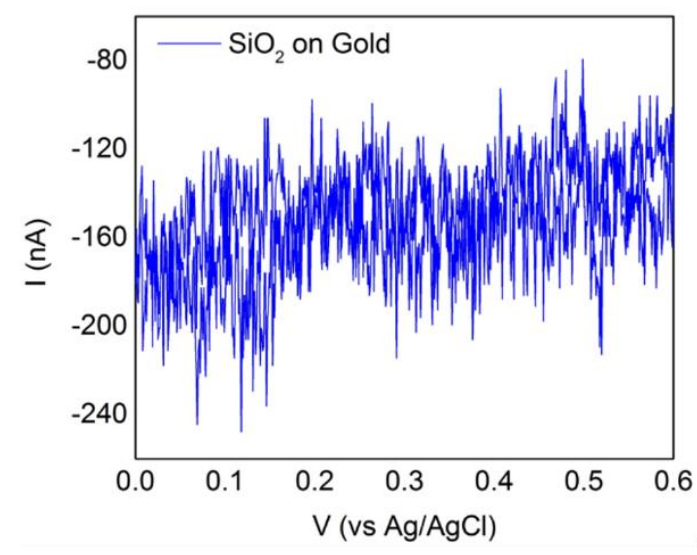

(a)

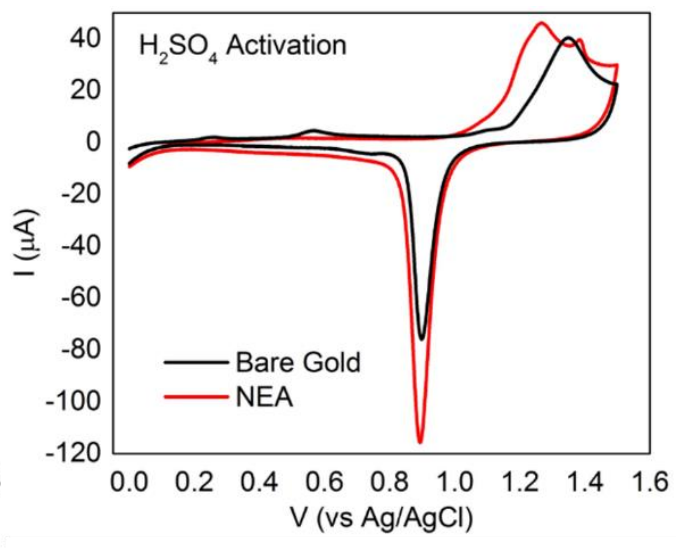

(b)

Figure 3.14: (a) Testing of the insulation layer and (b) $\mathrm{H}_{2} \mathrm{SO}_{4}$ activation of the electrodes.

\subsection{Results and Discussion}

\subsubsection{Cyclic voltammetry at Planar Gold Macroelectrode}

In this section, the results obtained from all the electrochemical tests will be analyzed. The changes in the signal from the changes in electrode dimensions in the NEAs will be examined and the performance will be relatively compared with a planar gold macroelectrode. As mentioned in the previous section, all the electrochemical analyses have been conducted in a PBS solution with $\mathrm{Fe}^{3+} / \mathrm{Fe}^{2+}$ redox couple.

Cyclic voltammograms are usually characterized by the positions of the forward and reverse peak currents, the ratio of the forward and peak currents, and the dependence of the magnitude of the peak currents on the scan rate. Figure 3.15 (a) shows the cyclic voltammogram of a planar bare gold macroelectrode of an area $0.0225 \mathrm{~cm}^{2}$ from the scan rates of $10 \mathrm{mV} / \mathrm{s}, 25 \mathrm{mV} / \mathrm{s}, 50 \mathrm{mV} / \mathrm{s}, 100$ $\mathrm{mV} / \mathrm{s}$ and $500 \mathrm{mV} / \mathrm{s}$ in a $\mathrm{Fe}^{2+} / \mathrm{Fe}^{3+}$ system. 


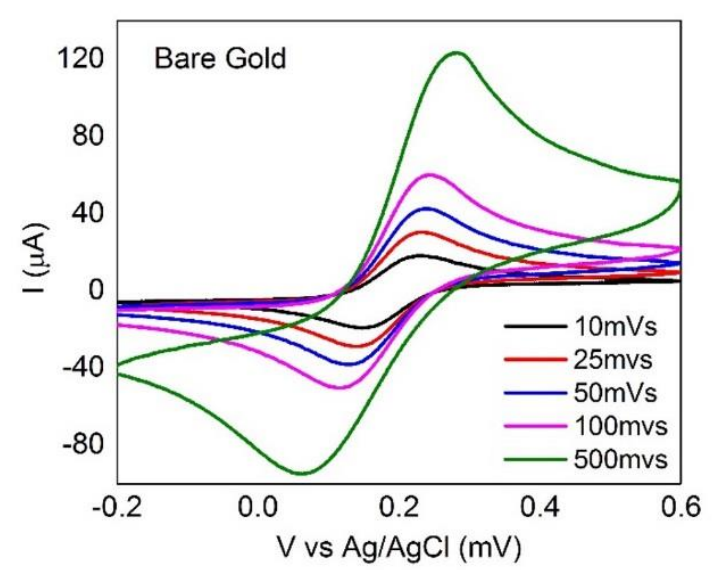

(a)

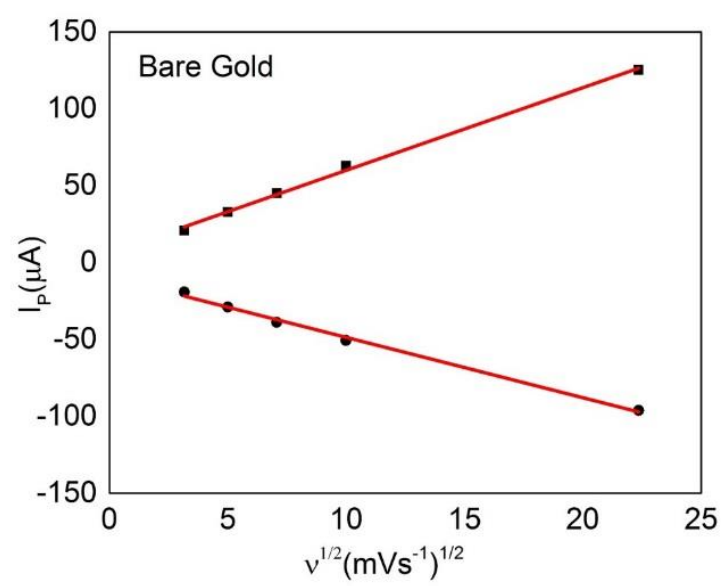

(b)

Figure 3.15: (a) CV plots and (b) linear fit for square root of scan rate vs. peak currents plot for the planar gold macroelectrode.

There are three things to be noticed. Firstly, the forward and reverse peak currents are almost equal i.e. the ratio of the peak currents is almost unity. Secondly, in the Figure 3.15 (b), the plot shows that there is a linear increase in the peak current values (both anodic and cathodic) with respect to square root of the scan rate. This is called Randles-Sevcik relation (eqn 2.13). And third is that there is a little difference between the oxidation and reduction potentials at all the scan rates, esp. under $100 \mathrm{mV} / \mathrm{s}$. All these factors contribute to show the reversibility of the system. Also the linear increase of peak currents with square root of scan rate exemplifies the mass transport dependence of the system.

In cyclic voltammetry, change in scan rate provides a path way to control the mass transport or electron transfer rate in an electrochemical process. There is always a tug-of-war between the diffusional transport of analytes and the faradaic electron transfer reaction at the electrode. At slow scan rates, the diffusion layer is thick and at faster scan rates the diffusion layer is thin. Usually at faster scan rates, the reaction at the electrode happens faster than the analyte's ability to reach the 
surface and hence the process is controlled by electron transfer rate. This is the cause for drift in peak potentials at higher scan rates. This is observed in the Figure 3.15 (a) at scan rate $500 \mathrm{mV} / \mathrm{s}$. At lower scan rates, the current peaks do not drift apart much, which shows the system reversibility.

Formal potential or standard potential $\left(\mathrm{E}_{\mathrm{O}}\right)$ is a characteristic value of a redox species which is similar to a characteristic wavelength peak of a species in spectroscopic studies. It is found by averaging the forward and reverse peak potentials and is usually independent of the scan rate for fast electron transfer processes. Slower scan rates should be used to determine Eo to minimize separation between the peaks. There is a lower limit of this scan rate which is set by the ability to maintain convection free conditions. Formal potentials calculated from the scan rates above $1 \mathrm{mV} / \mathrm{s}$ are usually considered useful ${ }^{[85]}$. The value $E_{O}$ is also used to calculate the electron transfer rate constant $(\mathrm{k})$ for the redox reaction.

As all the experiments were conducted in an unstirred solution, diffusion is the only process by which the analytes can reach the surface of the electrode. As the diffusion is slow and current does not increase further a point and instead it recedes, in macroelectrodes, once the depletion layer is formed. Hence, the peak shaped curves are produced, as shown in the Figure 3.15 (a). This is a characteristic property of linear or planar diffusion. The reversibility in the bare gold electrodes also shows that the electron transfer rate is faster than mass transport of the analytes i.e. $\mathrm{k}_{0}>>$ masstrans. In the quasi reversible and irreversible systems, finding electron transfer rates becomes more complex. For such complex systems, Nicolson and Shain have found out a way to find the approximate values of electron rate constants from the difference between the peak potentials ${ }^{[86]}$. Table 1 below shows the values of forward and reverse peak currents and voltages, 
formal potentials, peak separations and peak current ratios at different scan rates for planar gold macroelectrode.

Table 1: CV parameters of the planar gold macroelectrode.

\begin{tabular}{|c|c|c|c|c|c|c|c|}
\hline $\begin{array}{c}\text { Scan } \\
\text { Rate } \\
(\mathrm{v})\end{array}$ & $\begin{array}{c}\text { Forward } \\
\text { Peak Voltage } \\
\left(\mathrm{V}_{\mathrm{PF}}\right)\end{array}$ & $\begin{array}{c}\text { Reverse } \\
\text { Peak Voltage } \\
\left(\mathrm{V}_{\mathrm{PR}}\right)\end{array}$ & $\begin{array}{c}\text { Formal } \\
\text { Potential } \\
\left(\mathrm{E}_{\mathrm{O}}\right)\end{array}$ & $\begin{array}{c}\text { Peak } \\
\text { Separation } \\
\left(\Delta \mathrm{E}_{\mathrm{P}}\right)\end{array}$ & $\begin{array}{c}\text { Forward } \\
\text { Peak Current } \\
\left(\mathrm{I}_{\mathrm{PF}}\right)\end{array}$ & $\begin{array}{c}\text { Reverse } \\
\text { Peak Current } \\
\left(\mathrm{I}_{\mathrm{PR}}\right)\end{array}$ & $\begin{array}{c}\text { Peak } \\
\text { Current Ratio } \\
\left(\mathrm{I}_{\mathrm{PF}} / \mathrm{I}_{\mathrm{PR}}\right)\end{array}$ \\
\hline 10 & 225.96 & 149.61 & 187.785 & 76.35 & 20.87 & -19.24 & 1.08 \\
\hline 25 & 230.38 & 140.77 & 185.575 & 89.61 & 32.79 & -28.94 & 1.13 \\
\hline 50 & 236.15 & 130.58 & 183.365 & 105.57 & 45.11 & -38.84 & 1.16 \\
\hline 100 & 242.11 & 115.77 & 178.94 & 126.34 & 62.84 & -50.74 & 1.24 \\
\hline 500 & 278.88 & 65.96 & 172.42 & 212.92 & 125.2 & -96.08 & 1.36 \\
\hline
\end{tabular}

\subsubsection{CV at Varying Inter Electrode Spacing of NEA}

Based on the analysis of the planar gold macroelectrode, a comparison study of electrochemical responses of the NEAs with varying inter electrode spacing is done. Figure 3.16 shows the CV plots of the NEAs in comparison with planar gold macroelectrode. NEAs with the electrode diameter $100 \mathrm{~nm}$ and increasing inter electrode spacing of $200 \mathrm{~nm}, 500 \mathrm{~nm}$ and $1000 \mathrm{~nm}$ with scan rates of $10 \mathrm{mV} / \mathrm{s}, 25 \mathrm{mV} / \mathrm{s}, 50 \mathrm{mV} / \mathrm{s}, 100 \mathrm{mV} / \mathrm{s}$ and $500 \mathrm{mV} / \mathrm{s}$ are shown. It can be clearly observed that, at all the scan rates, as the distance between the nanoelectrodes increases the peak shaped attribute of the linear diffusion decreases and a steady state signal is attained. This is a strong indication of realization of diffusional domain independence which leads to a steady supply of analytes at the electrode surface. 

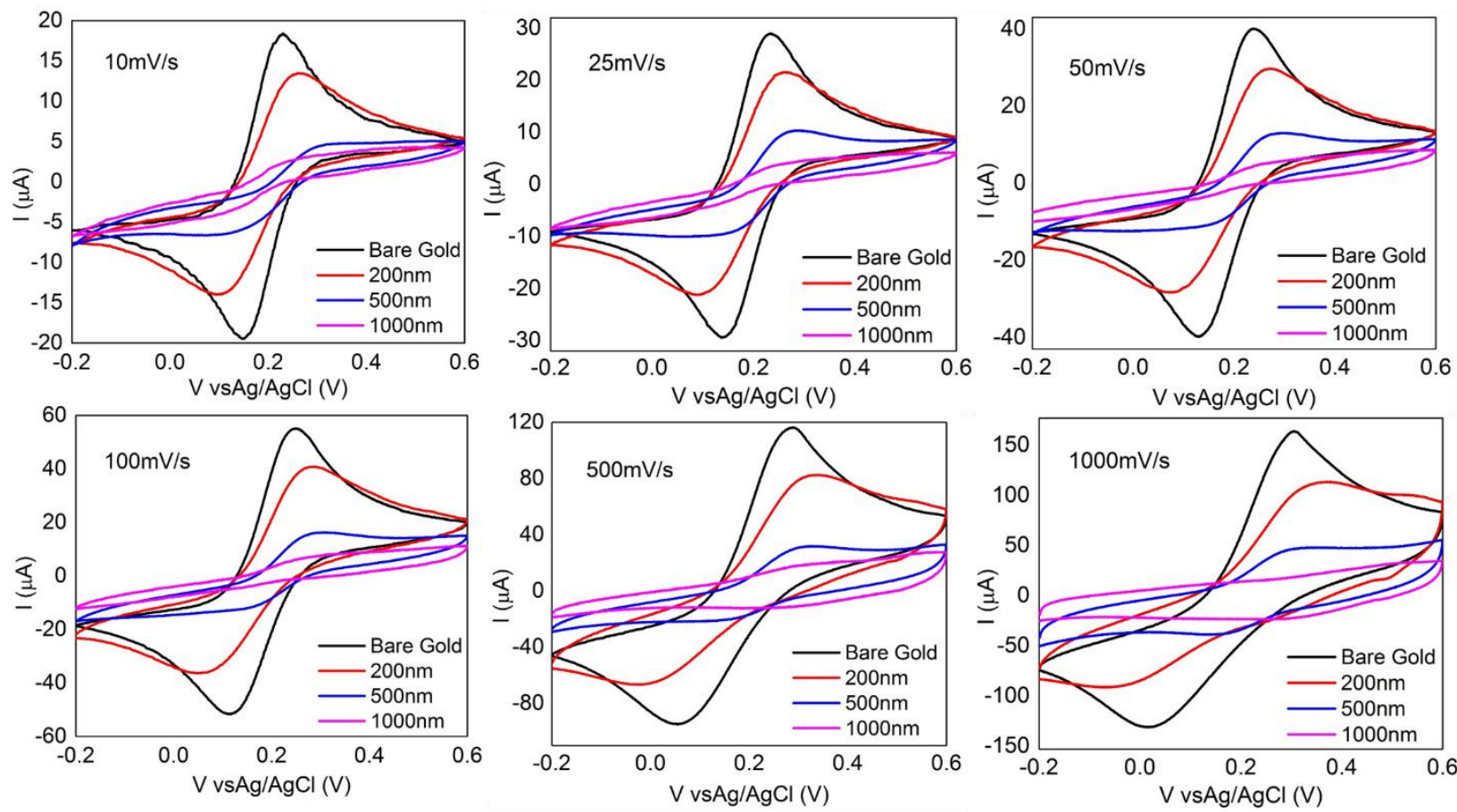

Figure 3.16: Comparative plots of NEAs with varying inter electrode spacing (x) at scan rates of $10 \mathrm{mV} / \mathrm{s}, 25 \mathrm{mV} / \mathrm{s}, 50 \mathrm{mV} / \mathrm{s}, 100 \mathrm{mV} / \mathrm{s}$ and $500 \mathrm{mV} / \mathrm{s}$; and a constant electrode diameter (d) of $100 \mathrm{~nm}$

In this study, the maximum distance between electrodes has been kept to be $1000 \mathrm{~nm}$ with electrode radius $50 \mathrm{~nm}$. Previous studies ${ }^{[87,88]}$ have shown that an interspacing distance between the electrodes greater than 10 times the radius of the electrodes leads to diffusional independence. In this study, the ratio of the distance between electrodes and the electrode radius is kept to be 20 .

\subsubsection{CV at Varying Electrode Diameter of NEA}

Figure 3.17 shows the CVs of NEAs with a constant inter electrode spacing of $1000 \mathrm{~nm}$ and varying electrode diameter of $100 \mathrm{~nm}, 400 \mathrm{~nm}$ and $700 \mathrm{~nm}$ with scan rates $10 \mathrm{mV} / \mathrm{s}, 25 \mathrm{mv} / \mathrm{s}$, $50 \mathrm{mv} / \mathrm{s}, 100 \mathrm{mV} / \mathrm{s}$ and $500 \mathrm{mV} / \mathrm{s}$. With a constant inter spacing and increasing electrode diameter, it is observed that the distance between the edges of the electrode is lessened and the electrodes 
become closer. This will have an impact on the diffusional domain of individual electrodes. As the diameter of the electrode increases, the diffusional domains of the individual electrode overlap and diffusion profile changes eventually from radial to linear. Also the increment in the diameter leads to more linear diffusion at the center of the electrode. This leads to a non-transient signal and the electrode behaves like a macroelectrode at longer times.
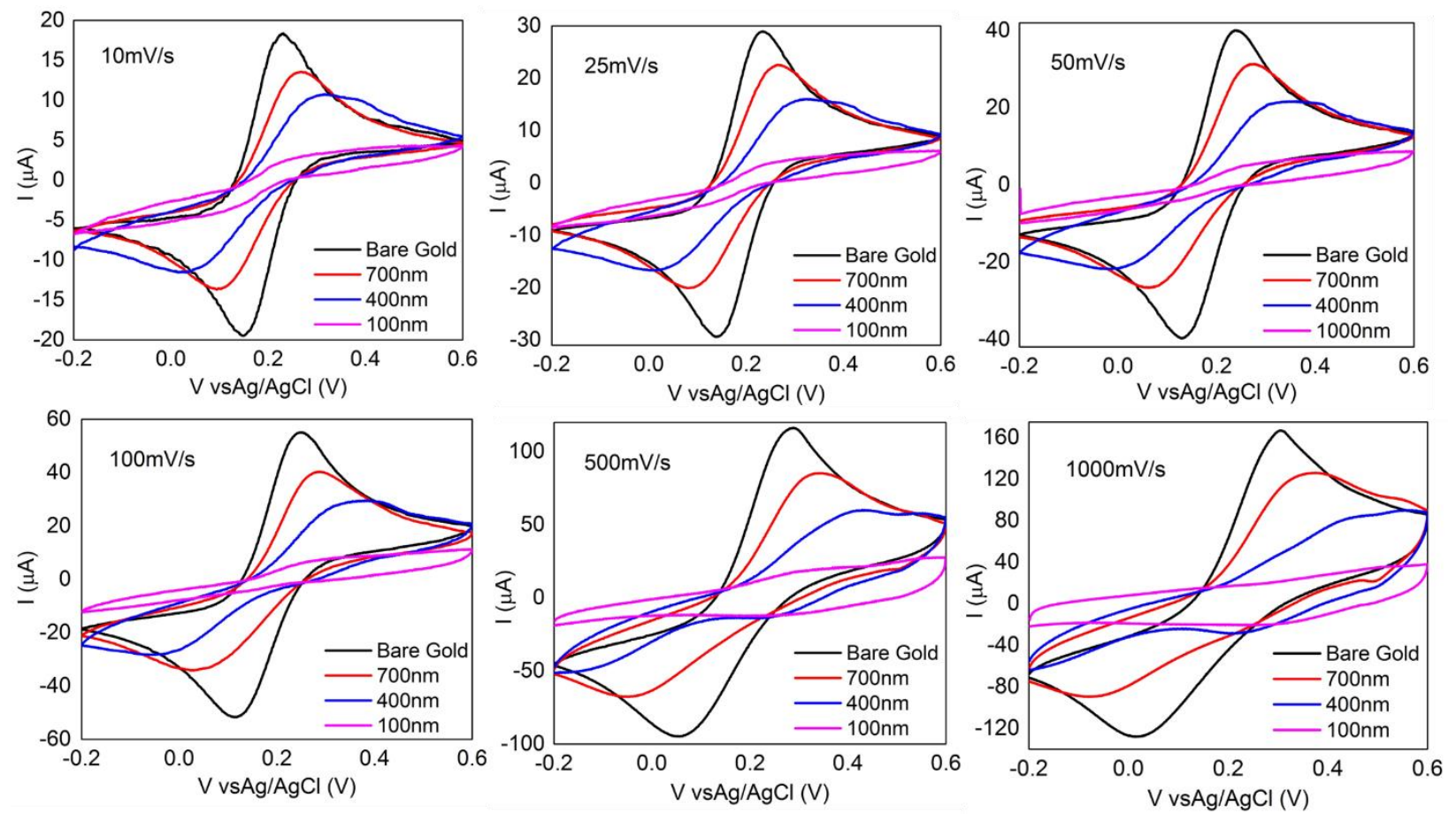

Figure 3.17: Comparative plots of NEAs with varying electrode diameter (d) at scan rates of 10 $\mathrm{mV} / \mathrm{s}, 25 \mathrm{mV} / \mathrm{s}, 50 \mathrm{mV} / \mathrm{s}, 100 \mathrm{mV} / \mathrm{s}$ and $500 \mathrm{mV} / \mathrm{s}$; and a constant inter electrode distance of $1000 \mathrm{~nm}$.

This is clearly seen in the Figure 3.17. As the diameter increases from $100 \mathrm{~nm}$ to $700 \mathrm{~nm}$, the signal is observed more like a planar gold macroelectrode with peak shaped curve. At extremely small diameter $(100 \mathrm{~nm})$ and due to the circular shape of the electrode at the edges, the diffusion 
is largely radial and there is less contribution from linear mode of diffusion. This helps in attaining steady state currents and making the diffusion profile independent of time.

\subsubsection{Optimized NEA Geometry}

From the analysis done in previous sections with varying inter electrode spacing and electrode diameters, an optimized electrode geometry for enhanced electrochemical sensitivity needs to be chosen. Obviously, the chosen electrode geometry needs to display a superior mass transport ability and high electron transfer rate. From the various dimensions fabricated above, it is evident that the NEA with an electrode diameter of $100 \mathrm{~nm}$ and an inter electrode spacing of $1000 \mathrm{~nm}$ has an excellent possibility of achieving steady state currents due to better mass transport and electron transfer rates.

Figure 3.18 shows the scan rate variation plot of the NEA with $d=100 \mathrm{~nm}$ and $\mathrm{x}=1000 \mathrm{~nm}$. The plot shows the steady state currents of the electrode with increasing scan rates. It has to be noted

that usually nanoelectrodes display current ranges in nano amperes ${ }^{[21,7]}$. But due to parallelization of individual nanoelectrodes, using the fabrication method stated in this chapter, the aggregated signal from all the individual electrodes falls in the range of micro amperes. This greatly boosts the electrochemical detection capabilities of the electrode system. 


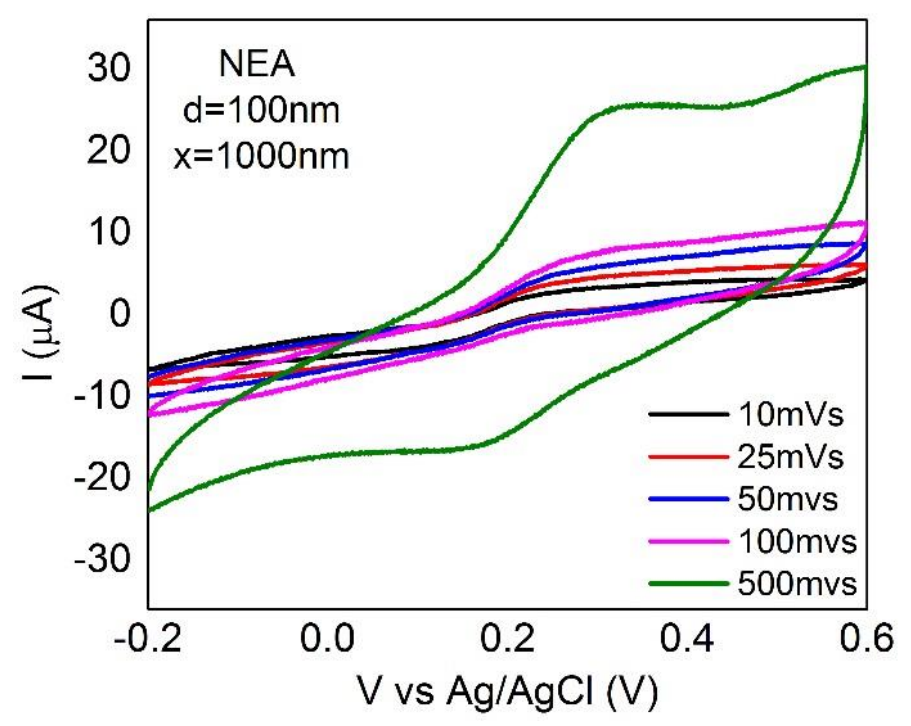

Figure 3.18: Scan rate variation plot of NEA with $d=100 \mathrm{~nm}$ and $\mathrm{x}=1000 \mathrm{~nm}$.

One important thing to be observed is the presence of charging current in the voltammograms. The observed charging currents of NEA is the sum of charging currents of the individual electrodes and is very low when compared to the planar macroelectrode. The sensitivity of the NEAs can be enhanced by reducing the charging currents further. One such method to decrease the net capacitance is to increase the thickness of the insulating layer. The insulating layer $\left(\mathrm{SiO}_{2}\right.$ layer $)$, ions in the electrolyte and the electrode act as a parallel plate capacitor. The fabricated NEA is an array of recessed disc electrode i.e. the electrode is receded into the insulating layer. As seen in the Figure 3.19, the diffusion profile at recessed electrode is very different from usual inlaid electrode. The diffusion of the analytes greatly depends in the length of the recession. So thicker insulating layer increases the distance of the electrode from the surface, which greatly hampers the diffusion of the analytes to the electrode surface and reduces the faradaic signal. So an optimum insulating thickness has to be used for fabrication. 
Inlaid disc electrode

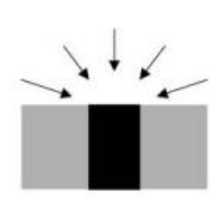

$$
I_{\lim }=4 n F D c^{b} r
$$

Recessed disc electrode

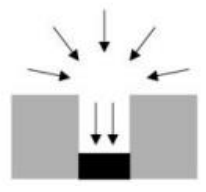

$$
I_{\lim }=\frac{4 \pi n F c^{b} D r^{2}}{4 L+\pi r}
$$

Figure 3.19: An image of diffusion profiles at inlaid and recessed electrodes ${ }^{[11]}$.

Table 2 below shows the values of forward and reverse peak currents and voltages, formal potentials, peak separations and peak current ratios at different scan rates for planar bare gold electrode.

Table 2: CV parameters of NEA

\begin{tabular}{|c|c|c|c|c|c|c|c|}
\hline $\begin{array}{c}\text { Scan } \\
\text { Rate } \\
(\mathrm{v})\end{array}$ & $\begin{array}{c}\text { Forward } \\
\text { Peak Voltage } \\
\left(\mathrm{V}_{\mathrm{PF}}\right)(\mathrm{mV})\end{array}$ & $\begin{array}{c}\text { Reverse } \\
\text { Peak Voltage } \\
\left(\mathrm{V}_{\mathrm{PR}}\right)(\mathrm{mV})\end{array}$ & $\begin{array}{c}\text { Formal } \\
\text { Potential } \\
\left(\mathrm{E}^{\mathrm{O}}\right)(\mathrm{mV})\end{array}$ & $\begin{array}{c}\text { Peak } \\
\text { Separation } \\
\left(\Delta \mathrm{E}_{\mathrm{P}}\right)(\mathrm{mV})\end{array}$ & $\begin{array}{c}\text { Forward } \\
\text { Peak Current } \\
\left(\mathrm{I}_{\mathrm{PF}}\right)(\mu \mathrm{A})\end{array}$ & $\begin{array}{c}\text { Reverse } \\
\text { Peak Current } \\
\left(\mathrm{I}_{\mathrm{PR}}\right)(\mu \mathrm{A})\end{array}$ & $\begin{array}{c}\text { Peak } \\
\text { Current Ratio } \\
\left(\mathrm{I}_{\mathrm{PF}} / \mathrm{I}_{\mathrm{PR}}\right)\end{array}$ \\
\hline 10 & 234.0 & 108.0 & 171.0 & 126 & 2.17 & -3.06 & 0.71 \\
\hline 25 & 268.0 & 76.2 & 172.1 & 191.8 & 2.79 & -3.03 & 0.92 \\
\hline 50 & 255.2 & 106.9 & 181.05 & 148.3 & 4.20 & -2.61 & 1.61 \\
\hline 100 & 321.0 & 108.4 & 214.7 & 212.6 & 7.89 & -3.86 & 2.04 \\
\hline 500 & 331.0 & $154 . .0$ & 242.5 & 177 & 14.39 & -4.12 & 10.27 \\
\hline
\end{tabular}

Figure 3.20 shows a peak current dependence on the square root of scan rate for the optimized NEA geometry and planar gold macroelectrode. It very evident that the NEAs shows a linear dependence on the square root of the scan rate i.e. increasing current due to better mass transport of the analytes. The linear dependency also shows that constant current function (the slope) which shows the fast electron transfer rates predicted by Nernst equation. 


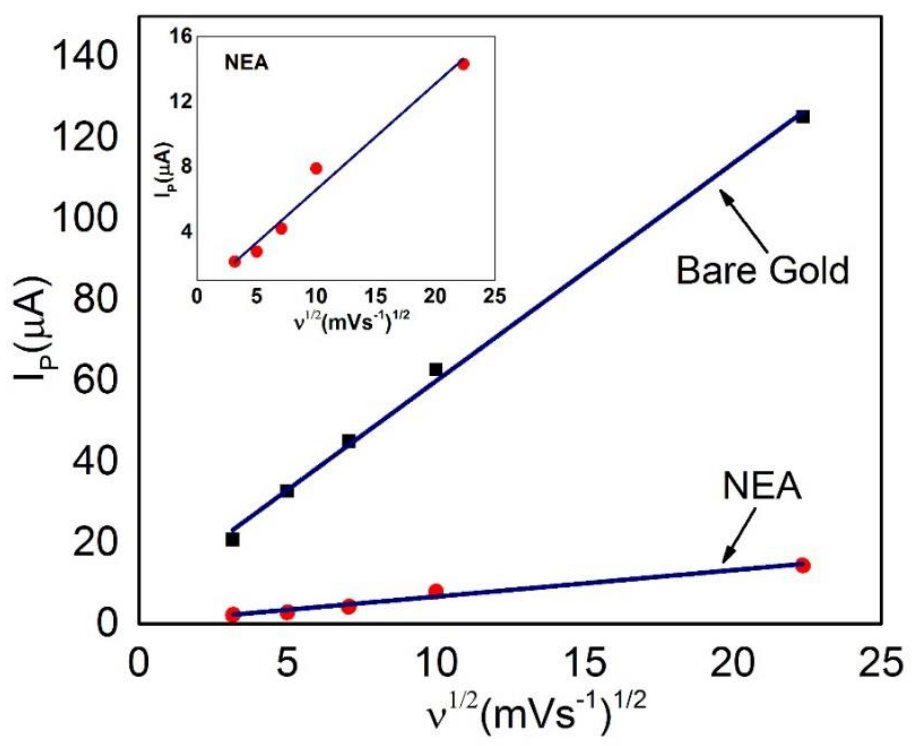

Figure 3.20: Peak current vs. the square root of the scan rate plot of NEA and planar gold macroelectrode.

\subsection{Conclusions}

In this chapter, the electrochemical advantages of a nanoelectrode over a macroelectrode has been shown. NEAs with varying inter electrode spacing and varying electrode diameters clearly show the electrochemical advantages of making the electrode smaller and farther in an array. Though the signal is smaller from the nano electrodes when compared to a planar gold macroelectrode, the NEAs will have a better mass transport and faster electron transfer rates leading to a better detection capability. This advantage is used to utilize the NEA as a biological sensor for detection of dopamine in biological levels of ascorbic acid in comparison with bare gold electrode in the next chapter. 


\section{Chapter 4: Nanoelectrode Array based Detection of Dopamine in Ascorbic Acid}

\subsection{Introduction}

Dopamine (DA) is neurotransmitter belonging to catecholamine family and is critically important to humans. It is the most abundant catecholamine involved in cerebral-body system and is produced by adrenal glands and several parts of the brain. Any changes in its biological levels has an direct effect on the neural system, brain-body coordination, organizational and control behavior. DA is also plays an important role in attention span, learning, and memory ${ }^{[89-92]}$. It also affects a variety of reward or pleasure perceiving behavior, cardiovascular and renal systems ${ }^{[93-95]}$. Low levels of dopamine in the central nervous system is a major cause of several neurological diseases such as schizophrenia, Parkinson's disease, and ADHD ${ }^{[96-99]}$.

In 1957, Carlson and this team first described the presence of dopamine in the brain ${ }^{[100]}$. Better understating of relationship between central nervous system, cognitive, emotional and behavioral state of humans have been made. Abnormal change in neurotransmitters have been linked to several psychological and physiological conditions such as depression ${ }^{[101]}$, drug dependence, ${ }^{[102]}$ schizophrenia, ${ }^{[103]}$ and degenerative diseases ${ }^{[104]}$ etc. Hence, monitoring the changes in such neurotransmitters is extremely important. Measurements with high selectivity, temporal resolution, spatial resolution are required and the materials need to be biocompatible. Due to advancement made in electronics and understanding in analytical chemistry, major progress has been made in making such measurements. But the procedures used to measure these neural transmitters are still limited in their ability to accurately measure the changes that occur in central nervous system. 


\subsection{Literature Review}

Most common techniques used for the measurement of neurotransmitters are electrochemical analysis using biocompatible electrodes, liquid chromatography (LC), capillary electrophoresis (CE), and mass spectrometry (MS). Microelectrodes and biosensors can show high temporal and spatial resolution but only a few the neurotransmitters can be detected by direct redox reaction at an electrode ${ }^{[105]}$. Measurement of the electroactive neurotransmitters is usually complicated due to interference from relatively high concentration of other electroactive metabolites such as ascorbic acid. Various other reasons can also effect the detection such as electrode fouling. Also, usually electrochemical detection may require long periods for sample accumulation time, which limits the length and monitoring ability.

Different types of micro and nanoelectrodes have been produced to electrochemically detect dopamine in presence of other biological metabolites. Biocompatible materials such gold, platinum, and carbon based materials are commonly used to prepare the electrodes. Carbon based electrodes have an excellent compatibility with biological materials, allowing for a wide range of modifications. Among the carbon-based electrode, boron-doped diamond electrode ${ }^{[106,107]}$ and surface modified glassy carbon electrodes ${ }^{[108-110]}$ have been widely used. Using microfabrication methods, carbon inter digitated electrodes were used with a LOD of $1.5 \mu \mathrm{M}{ }^{[111]}$. Another biocompatible material, gold has been extensively used for detection of DA. Gold nanoelectrodes such nanoparticle arrays ${ }^{[112]}$, nanowires ${ }^{[113]}$, nanoporous ${ }^{[114]}$, enzyme coated ${ }^{[115]}$, carbon nanotube coated ${ }^{[116,117]}$, self-assembled monolayer coated ${ }^{[118]}$ etc. have been used to enhance the detection capabilities of the gold electrodes for DA detection. Blake et. al. had created a gold nanostructured gold array using electrodeposition and analyzed the difference between a gold 
macroelectrode and gold nano array structure electrode for DA detection [119]. They showed that the nanostructure containing more surface area had more active sites showing enhanced electrochemical activity compared to a planar gold macroelectrode. Electrodes were prepared using graphene-Pt nano composites with detection levels of $30 \mathrm{nM}$ of $\mathrm{DA}^{[120]}$. Using carbon fiber on glass electrodes, a real time in-situ analysis of changes in dopamine were recorded in the rat brain after inducing it with cocaine ${ }^{[121]}$. Microwell arrays and nanoelectrode arrays of different materials have also been used for DA detection. Plane and recessed geometry gold electrodes have been fabricated to enhance the detection through redox cycling ${ }^{[122,123]}$. The advantages of microelectrode array and nanoelectrode array have also been utilized. In-plane gold microelectrodes were prepaid using chemical mechanical planarization with a LOD of $11 \mathrm{~nm}^{[124]}$. No significant electrode fouling was recorded at $100 \mathrm{~nm}$ for DA for 4 hours of amperometric studies. A recessed ring-disk gold nano electrode structure was created for nanofluidic channel and analyzing the analytes with redox cycling ${ }^{[125]} .20 \mathrm{nM}$ of LOD was detected for DA.

\subsection{Experimental Section}

\subsubsection{Materials}

Following materials have been used for electrochemical testing: dopamine hydrochloride (Sigma Aldrich, USA), 1-ascorbic acid (Amresco Inc., USA), phosphate buffer saline tablets (Sigma Aldrich, USA) and deionized water produced by the Milli-Q Integral 3/5/10/15 system (18.2 $\mathrm{M} \Omega \cdot \mathrm{cm}$, Millipore Corp., USA). 


\subsubsection{Instruments}

Following instruments have been used for electrochemical testing: Gamry reference 600 potentiostat and Gamry echem framework software (Gamry Instruments Inc., USA) for signal recording, BASi C3 cell stand (BASi Inc., USA) for electrochemical analysis.

\subsubsection{Electrochemical Testing}

Similar set up described in Chapter 3 has been utilized for the dopamine detection. The nanoelectrode array with $\mathrm{d}=100 \mathrm{~nm}$ and $\mathrm{x}=1000 \mathrm{~nm}$ and planar bare gold macroelectrode were used for electrochemical testing. The electrodes were electrochemically activated in $0.5 \mathrm{M} \mathrm{H}_{2} \mathrm{SO}_{4}$ as in the Chapter 3. Differential pulse voltammetry (DPV) has been utilized to detect the DA and AA in PBS. In this technique, the sensitivity in the voltammogram is improved by reducing the charging current by delaying the recording of current signal as late as possible after application of the potential step (or pulse). All the experiments are conducted in phosphate buffer saline solution from $-0.2 \mathrm{~V}$ to $+0.6 \mathrm{~V}$ with following parameters for DPV. Step size: $5 \mathrm{mv}$, sample period: $1 \mathrm{sec}$, pulse time: $0.04 \mathrm{sec}$ and pulse size: $20 \mathrm{mV}$. The parameters have been optimized for a better full width half maximum (FWHM) of the signal.

\subsection{Results and Analysis}

\subsubsection{DA Detection with NEA}

The physiological levels of DA change from $10 \mathrm{nM}$ to $1 \mu \mathrm{M}$ in human body and ascorbic acid (AA) is usually 100 to 1000 times more than DA. The biggest challenge to detect DA is that the redox potentials of the DA and AA are too close and usually difficult to differentiate through electrochemical analysis. Special instrumentation or procedures are to be considered for successful determination of both the species simultaneously. Electrodes with better sensitivity are required 
for simultaneous detection. Since the electrochemical tests have proved in Chapter 3 that the NEA shows better mass transport and faster kinetics, the NEA is tested for DA detection with their advantageous capabilities over planar macroelectrode.

Figure 4.1 shows the DPV plot for NEA in PBS solution from $-0.2 \mathrm{~V}$ to $0.6 \mathrm{~V}$. A peak signal can be observed from PBS at around $165 \mathrm{mV}$ and this is considered to be the background signal. Figure 4.1 (b) is the plot for DPV with increasing concentration levels of ascorbic acid (AA) (10 $\mu \mathrm{M}, 50 \mu \mathrm{M}$ and $500 \mu \mathrm{M}$ ) in PBS solution. It can be observed from the plot that ascorbic acid has distinctive peak at around $-55 \mathrm{mV}$ and, interestingly, there is no signal observed at lower concentrations at $10 \mu \mathrm{M}$ and $50 \mu \mathrm{M}$.

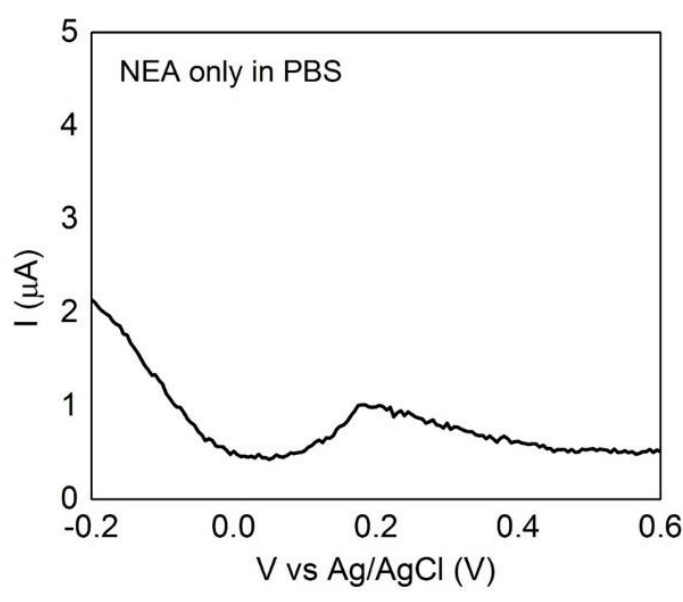

(a)

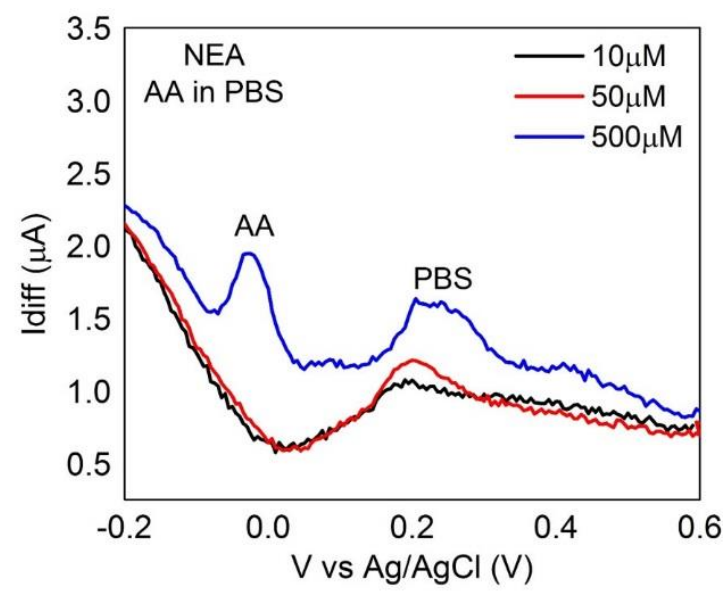

(b)

Figure 4.1: (a) DPV plots of NEA in PBS only and (b) DPV plot of NEA in increasing concentrations of ascorbic acid $(10 \mu \mathrm{M}, 50 \mu \mathrm{M}$ and $500 \mu \mathrm{M})$ in PBS. 
Now, a comparative experiment between the planar gold macroelectrode and NEA is conducted for detection of dopamine in presence of ascorbic acid. In the below described experiments, all the concentration levels of dopamine are detected in presence of $500 \mu \mathrm{M}$ ascorbic acid and in PBS solution. Figure 4.2 (a) shows the DPV plots of planar gold macroelectrode with increasing concentrations of $100 \mathrm{nM}, 500 \mathrm{nM}, 1 \mu \mathrm{M}, 5 \mu \mathrm{M}$, $10 \mu \mathrm{M}$ and $50 \mu \mathrm{M} \mathrm{DA}$ in $500 \mu \mathrm{M}$ AA in PBS solution. The figure shows the increasing peak intensity with increasing DA concentrations levels. It is to be observed that the planar electrode fails to differentiate the peak currents of dopamine and ascorbic acid. Signal from both the analytes are added and increasing peak currents is observed.

Figure 4.2 (b), shows the plot of DA detection in presence of AA at the NEA. A long range of $100 \mathrm{nM}, 500 \mathrm{nM}, 1 \mu \mathrm{M}, 5 \mu \mathrm{M}, 10 \mu \mathrm{M}, 20 \mu \mathrm{M}, 30 \mu \mathrm{M}, 40 \mu \mathrm{M}, 50 \mu \mathrm{M}, 80 \mu \mathrm{M}, 120 \mu \mathrm{M}, 160$ $\mu \mathrm{M}$, and $200 \mu \mathrm{M}$ of DA concentrations is utilized to observe the signal. It clearly noticeable the ability of NEA to differentiate the signal between the DA and AA. With increasing levels of DA, steady increase in signal is observed and signals from AA is observed to be constant. This ability to differentiate the AA and DA with increase in signal is attributed to an excellent mass transport of the analytes to the surface of the electrode. Lack of any peak shift shows the excellent electron transfer kinetics at the surface of the electrode. One thing to be observed is from lower concentrations to higher concentrations, there is shift in peak positions of DA. This is because at lower concentrations currents from PBS is dominating and as the DA concentrations increase the signal move towards the DA peak value at around $162 \mathrm{mV}$. 


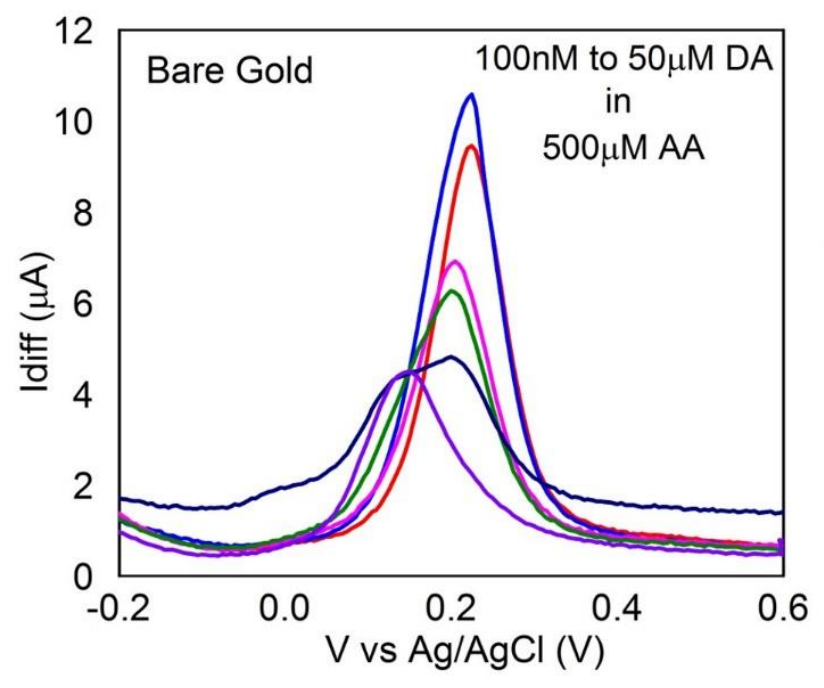

(a)

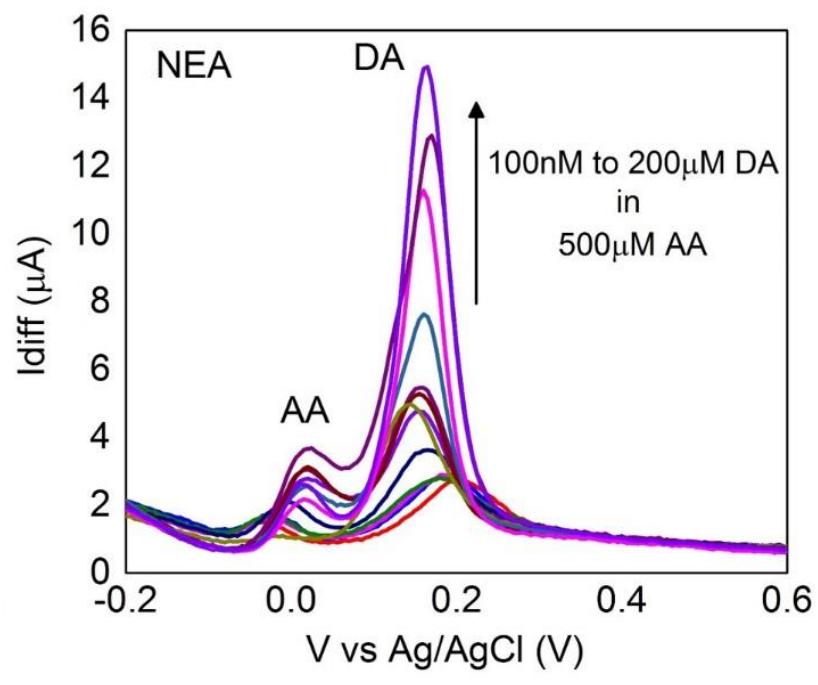

(b)

Figure 4.2: Concentration plots of (a) planar gold macroelectrode with increasing concentrations of DA from $100 \mathrm{nM}$ to $50 \mu \mathrm{M}$ in $500 \mu \mathrm{M}$ AA and (b) NEA with increasing concentrations of DA from $100 \mathrm{nM}$ to $200 \mu \mathrm{M}$ in $500 \mu \mathrm{M}$ AA.

One problem faced while the experiments were conducted was electrode fouling. Electrode fouling of the gold electrode was recorded at dopamine concentrations more than $10 \mu \mathrm{M}$ for both planar macroelectrode and nanoelectrode. From the repeatability tests, the signal from the electrode for macroelectrode and nanoelectrode were stable and were highly repeatable. But this is not the case for DA levels more than $10 \mu \mathrm{M}$. From DA concentrations of $10 \mu \mathrm{M}$ to $200 \mu \mathrm{M}$, the electrode had reduced signals in the repeatability tests and this is attributed to the affinity of the DA to adsorb on the surface of the gold ${ }^{[126]}$. This is phenomenon can be observed in the change in current peaks from Figure 4.3.

Increasing values of peak currents with respect to increasing values of DA concentration is shown in the Figure 4.3. Seen on the whole, the increment in the peak current values look rising linearly with increase in the DA. But when looked closely, the slope of the points after $20 \mu \mathrm{M}$ of DA 
reduces. This might be due to the observance of electrode fouling at concentration higher than $10 \mu \mathrm{M}$ as explained previously. The slopes of the data points from $20 \mu \mathrm{M}$ to $200 \mu \mathrm{M}$ is little less than the data slope of the points from $100 \mathrm{nM}$ to $10 \mu \mathrm{M}$ of DA. Hence, as seen in the fitting plot, two different linear fittings were used. From the DA concentration range, $100 \mathrm{nM}$ to $10 \mu \mathrm{M}$ a linear fit with slope 0.13 is recorded and from $20 \mu \mathrm{M}$ to $200 \mu \mathrm{M}$ a linear fit with a slope 0.06 is observed. Both the fittings have an $R^{2}$ value of more than 0.97 . This clearly shows the change in dopamine detection abilities of the NEA after $10 \mu \mathrm{M}$. There is a clear increase in the current observed for the DA concentration of $100 \mathrm{nM}$ when compared to the background current. Hence, limit of detection is found to be about $80 \mathrm{nM}$ of DA concentration.

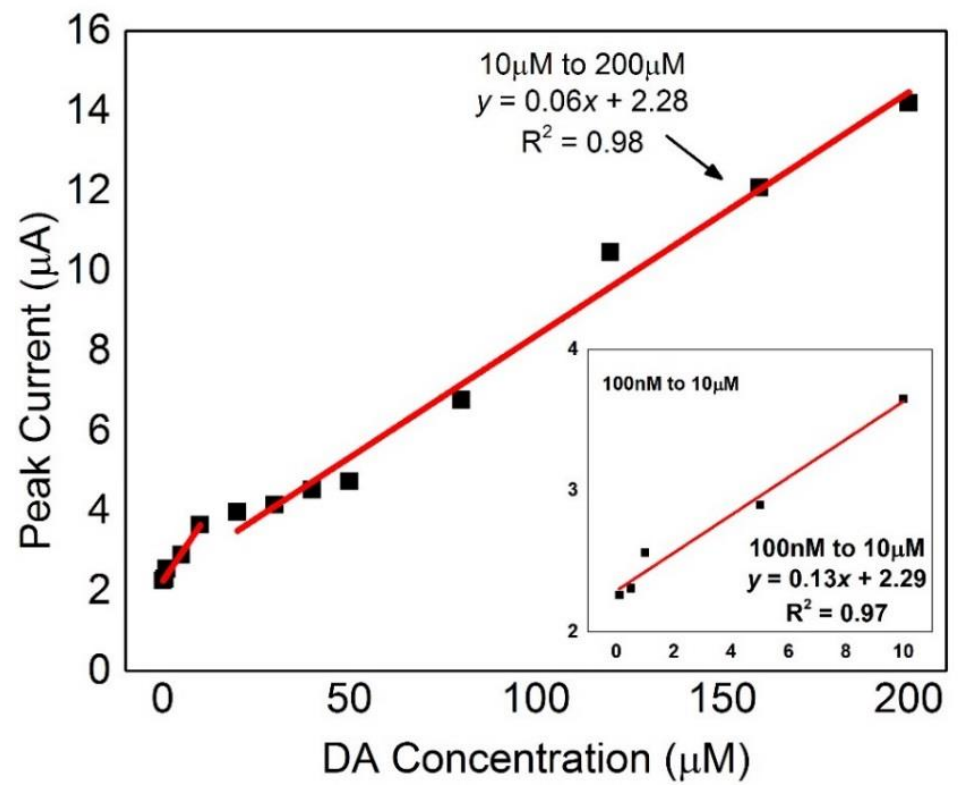

Figure 4.3: DA concentration vs. peak current plot for DPV of NEA 


\subsubsection{Repeatability}

The NEA electrodes were tested for repeatability and reliability. 6 separate experiments were conducted with DA concentrations of $500 \mathrm{nM}, 5 \mu \mathrm{M}$ and $50 \mu \mathrm{M}$ concentrations in $500 \mu \mathrm{M}$ AA in PBS solution. Figure 4.3, shows the overlapped plots of (a) $500 \mathrm{nM}$, (b) $5 \mu \mathrm{M}$ and (c) $50 \mu \mathrm{M}$ of DA tested separately. For the testing of electrodes with $50 \mu \mathrm{M}$, the electrode had to be activated with sulfuric acid cycling for every experiment.

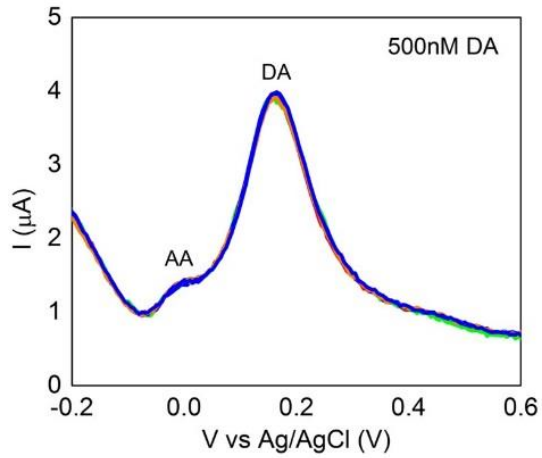

(a)

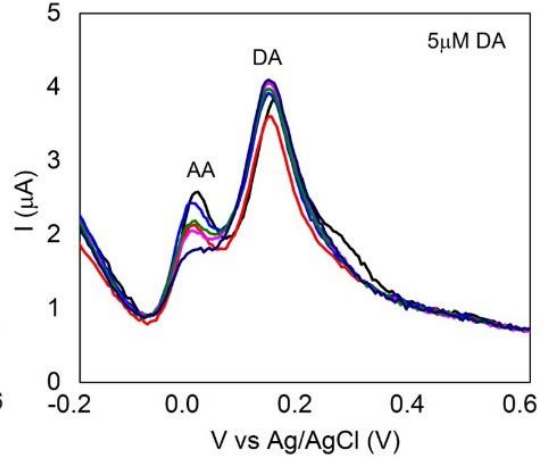

(b)

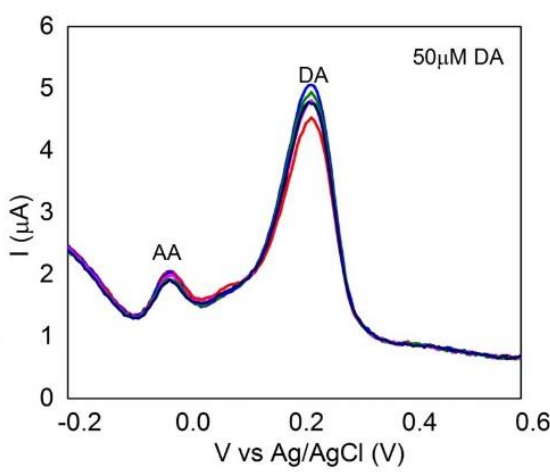

(c)

Figure 4.4: Repeatability test of NEA in $500 \mathrm{nM}, 5 \mu \mathrm{M}$ and $50 \mu \mathrm{M}$ DA in $500 \mu \mathrm{M}$ AA in PBS.

\subsection{Conclusion}

In this chapter, the excellent ability of the nanoelectrode array to act as a biosensor has been shown. The NEA has clearly distinguished and detected the peaks of dopamine and ascorbic acid is phosphate buffer saline solution showing an excellent selectivity. The gold planar macroelectrode has failed to differentiate the peaks of biomolecules. This is attributed to the excellent mass transport and faster kinetics at the surface of the nanoelectrodes when compared to the macro electrode. 


\section{Chapter 5: Conclusions and Future Work}

\subsection{Conclusions}

In this thesis, a new fabrication procedure for producing nanoelectrode array was developed using nanosphere lithography (NSL). The procedure had the ability to the control the distance between the electrode and the diameters of individual electrodes on a large area. The advantages of the electrochemistry at the nanoscale were studied in comparison with a planar bare gold macroelectrode. The variation of the electrochemistry signal with varying dimensions of the electrode were studied; and geometry was optimized for electrochemical analysis was determined to use it as a biological sensor. Below mentioned are some of the highlights of the work:

1) Large area nanoelectrode arrays with an excellent control on the periodicity and electrode diameter have been fabricated using nanosphere lithography. The varying inter electrode spacing of $200 \mathrm{~nm}, 500 \mathrm{~nm}$ and $1000 \mathrm{~nm}$ and electrode diameters of $100 \mathrm{~nm}, 400 \mathrm{~nm}$ and $700 \mathrm{~nm}$ were prepared with $\mathrm{SiO}_{2}$ as the insulating layer covering on plane gold electrode.

2) An inter electrode spacing of $1000 \mathrm{~nm}$ and electrode diameter of $100 \mathrm{~nm}$ is found to be the best geometry for electrochemical analysis. Unlike planar gold macroelectrode and other NEAs, steady-state currents were obtained at varied scan rates. This shows the steady supply of the analytes to nanoelectrode surface for longer times.

3) It has been experimentally shown, reducing the electrode size and increasing the distance between the electrodes lead to independence of diffusional domains at the electrode surface. The linear slope of the peak current vs. the square root of scan rate plot proves the better mass transport of analytes and faster electrode reaction kinetics at the NEA. 
4) NEA with optimized geometry was used as a biosensor for detection of dopamine (DA) in presence of high levels of ascorbic acid (AA). The planar gold macroelectrode has failed to distinguish and differentiate the oxidation peaks of DA and AA. On the other hand, the optimized NEA has shown an excellent ability to detect and differentiate the DA and AA signals. The detection had an almost linear range from $100 \mathrm{nM}$ to $200 \mu \mathrm{M}$.

5) Electrode fouling due to adsorption of DA on the gold electrode surface was detected for the DA levels more than $10 \mu \mathrm{M}$ for both the electrodes. Hence, current peaks with lower slopes were observed after DA levels of $10 \mu \mathrm{M}$ when compared to DA levels from $100 \mathrm{nM}$ to $10 \mu \mathrm{M}$.

6) NEAs have shown an excellent repeatability, however for DA detection above $10 \mu \mathrm{M}$ the electrodes had to be electrochemically activated. An excellent selectivity has been observed with the separation of peaks of DA and AA using NEA. Limit of detection is $80 \mathrm{nM}$.

\subsection{Recommendations on Future Work}

The electrochemical study of the NEA with varied dimensions has given formal guidelines for designing nanoelectrodes for enhanced detection abilities. Following implications can be made from this work presented:

1) The fabricated gold NEAs can be used in real time detection of biological and chemical species. Changes in hormones, neuro transmitters, parasites etc. as biological analytes and heavy metals, industrials chemical wastes as chemical analytes can be quantified in real time. 
2) Theoretical simulations based on the presented work can help design smaller electrodes with better detection capabilities. Protocols for designing better electrodes with radius smaller than $50 \mathrm{~nm}$ can be realized for extreme detection levels.

3) Reducing the size of the substrate and integration with techniques such as microfluidic devices will help make the sensors portable and readily available.

4) With variations in the fabrication process, electrodes with new shapes and sizes and materials can be realized to expand the horizons of the current technology for varied applications. 


\section{References}

1. Mark R. Wightman, Anal. Chem., 1981, 53, 1125A-1134A.

2. Martin Fleischmann, Jamal Ghoroghchian, Debra Rolison, and Stanley Pons, J. Phys. Chem., 1986, 90, 6392-6400.

3. Mark R. Wightman, Science, 1987, 240, 415-420.

4. Koichi Aoki, Electroanalysis, 1993, 5, 627-639.

5. Rosemary Feeney, Samuel Kounaves, Electroanalysis, 2000, 12, 677-684.

6. Cynthia G. Zoski, Electroanalysis, 2002, 14, 1041-1051.

7. Damien W. M. Arrigan, Analyst, 2004, 129, 1157-1165.

8. Xiaoyu Zhang, Alyson V. Whitney, Jing Zhao, Erin M. Hicks, and Richard P. Van Duyne, J. Nanosci. Nanotechnol., 2006, 6, 1-15.

9. John C. Hulteen and Richard P. Van Duyne, J. Vac. Sci. Technol.A, 1995, 13, 1553-1558.

10. Pierre Colson, Catherine Henrist, and Rudi Cloots, J. Nanomaterials, 2013.

11. Electrochemical Methods, $2^{\text {nd }}$ ed, Allen J. Bard, Larry R. Faulkner, 2001.

12. Analytical Electrochemistry, $3^{\text {rd }}$ ed., Joseph Wang, 2006.

13. Martin C. Henstridge and Richard G. Compton, The Chemical Record, 2012, 12, 63-71.

14. R. Mark Wightman, Anal. Chem., 1981, 53, 1125A

15. R. Mark Wightman, Science, 1988, 240, 415-420.

16. Koichi Aoki, Electroanalysis, 1993, 5, 627-639.

17. Karel Štulik, Christian Amatore, Karel Holub, Vladmir Marecek and Wlodzimierz Kutner, Pure Appl. Chem., 2000, 72, 1483-1492.

18. Cynthia G. Zoski, Electroanalysis, 2002, 14

19. Damien W. M. Arrigan, Analyst, 2004, 129, 1157-1165. 
20. Jonathan T. Cox and Bo Zhang, Annu. Rev. Anal. Chem., 2012, 5, 253-272.

21. Karen Dawson and Alan O’Riordan, Annu. Rev. Anal. Chem., 2014, 7, 163-181

22. Edmund J. F. Dickinson and Richard G. Compton, J. Phys. Chem. C, 2009, 113, 1758517589.

23. Christian Amatore, Cécile Pebay, Laurent Thouin, Aifang Wang, Echem. Comm., 2009, 11 $1269-1272$.

24. Trevor J. Davies, Richard G. Compton, J. Electroanal. Chem., 2005, 585, 63-82.

25. Cyclic Voltammetry Simulation at Microelectrode Arrays, Proceedings of the COMSOL Users Conference, 2006, Milano.

26. Francois G. Chevallier, Li Jiang, Timothy G.J. Jones, Richard G. Compton, J. Electroanal. Chem. 2006 , 587, 254-262.

27. Jidong Guo and Erno Lindner, Anal. Chem., 2009, 81, 130-138.

28. Hye Jin Lee, Carine Beriet, Rosaria Ferrigno, Hubert H. Girault, J. Electroanal. Chem. 2001, 502, 138-145.

29. Mairi E. Sandison, Natalie Anicet, Andrew Glidle, and Jonathan M. Cooper, Anal. Chem., 2002, 74, 5717-5725.

30. Neus Godino, Xavier Borrise, Francesc Xavier Munoz, Francisco Javier del Campo, and Richard G. Compton, J. Phys. Chem. C, 2009, 113, 11119-11125.

31. Denis Menshykau, Xing-Jiu Huang, Neil V. Rees, F. Javier del Campo, Francesc Xavier Munoz and Richard G. Compton, Analyst, 2009, 134, 343-348.

32. H. W. Deckman and J. H. Dunsmuir, Appl. Phys. Letters., 1982, 41, 377-379.

33. H. W. Deckman and J. H. Dunsmuir, J. Vac. Sci. Technol. E, 1988, 4, 1109-1112.

34. John C. Hulteen and Richard P. Van Duyne, J. Vac. Sci. Technol. A, 1995, 13, 1553-1558. 
35. M. Winzer, M. Kleiber, N. Dix, R. Wiesendanger, Appl. Phys. A, 1996, 63, 617-619.

36. Frank Burmeister,Claudia Schafle, Thomas Matthes, Matthias Bohmisch, Johannes Boneberg, and Paul Leiderer, Langmuir, 1997, 13, 2983-2987.

37. John C. Hulteen, David A. Treichel, Matthew T. Smith, Michelle L. Duval, Traci R. Jensen, and Richard P. Van Duyne, J. Phys. Chem. B, 1999, 103, 3854-3863.

38. Philip N. Bartlett, Peter R. Birkin and Mohamed A. Ghanem, Chem. Commun., 2000, 1671167.

39. Christy L. Haynes, Adam D. McFarland, Matthew T. Smith, John C. Hulteen, and Richard P. Van Duyne, J. Phys. Chem. B 2002, 106, 1898-1902.

40. Amanda J. Haes, Christy L. Haynes, Richard P. Van Duyne, Mat. Res. Soc. Symp., 636, D4.8.1 - D4.8.6.

41. Chiseki Haginoya, Masayoshi Ishibashi, and Kazuyuki Koike, Appl. Phys. Lett., 1997, 71.

42. Peng Jiang and Michael J. McFarland, J. Am. Chem. Soc., 2004, 126, 13778-13786.

43. Peng Jiang and Michael J. McFarland, J. Am. Chem. Soc. 2005, 127, 3710-3711.

44. J. Rybczynski, U. Ebels, M. Giersig, Colloids and Surfaces A: Physicochem. Eng. Aspects, 2013, 219, 1-6.

45. K. Kempa, B. Kimball, J. Rybczynski, Z. P. Huang, P. F. Wu, D. Steeves, M. Sennett, M. Giersig, D. V. G. L. N. Rao, D. L. Carnahan, D. Z. Wang, J. Y. Lao, W. Z. Li, and Z. F. Ren, Nano Lett., 2003, 3, 13-18.

46. Huaqing Li, Jeremy Low, Kolin S. Brown, and Nianqiang Wu, IEEE Sensors Journal, 2008, 8, 880-884.

47. A. Pockels, Nature, 1891 43, 437.

48. I. Langmuir, J. Am. Chem. Soc., 1917, 39, 1848. 
49. K.B. Blodgett, J. Am. Chem. Soc., 1935, 57, 1007.

50. Shaw, D.J., Introduction to Colloid and Surface Chemistry, Butterworth \& Co, London, 1980.

51. Laughlin, R.G. The Aqueous Phase Behaviour of Surfactants, Academic Press Inc., San Diego, 1994.

52. Kuniaki Nagayama, Colloids and Surfaces A, 1996, 109, 363-374.

53. Dimitris Stamou, Claus Duschl and Diethelm Johannsmann, Phys. Rev. E, 2000, 62, 52635272.

54. E. C. Mbamala and H. H. von Grünberg, Phys. Rev. E, 2003. 67, 1-11.

55. Elizabeth J. Tull, Philip N. Bartlett, and Kate R. Ryan, Langmuir, 2007, 23, 7859-7873.

56. F. Lenzmann, J K. Li,z A. H. Kitai, and H. D. H. Stover, Chem. Mater. 1994, 6, 156-159.

57. Christy L. Haynes and Richard P. Van Duyne, J. Phys. Chem. B, 2001, 105, 5599-5611.

58. Anjeanette D. Ormonde, Erin C. M. Hicks, Jimmy Castillo, and Richard P. Van Duyne, Langmuir, 2004, 20, 6927-6931.

59. Chun-Wen Kuo, Jau-Ye Shiu, and Peilin Chen, Chem. Mater., 2003, 15, 2917-2920.

60. Chun-Wen Kuo, Jau-Ye Shiu, Yi-Hong Cho, and Peilin Chen, Adv. Mater, 2003, 15, 10651068.

61. C L Cheung, R J Nikoli, C E Reinhard and T FWang, Nanotechnology, 2006, 17, 1339-1343.

62. Junghoon Yeom, Daniel Ratchford, Christopher R. Field, Todd H. Brintlinger , and Pehr E. Pehrsson, Adv. Funct. Mater. 2014, 24, 106-116.

63. B. J.-Y. Tan, C.-H. Sow, K.-Y. Lim, F.-C. Cheong, G.-L. Chong, A. T.-S. Wee, and C.-K. Ong, J. Phys. Chem. B, 2004, 108, 18575-18579.

64. Dae-Geun Choi, Hyung Kyun Yu, Se Gyu Jang, and Seung-Man Yang, J. Am. Chem Soc., 2004, 126, 7019-7025. 
65. A. Kosiorek, W. Kandulski, P. Chudzinski, K. Kempa, and M. Giersig, Nano Letters, 2004, 4, 1359-1363.

66. B. J. Y. Tan, C. H. Sow, T. S. Koh, K. C. Chin, A. T. S. Wee, and C. K. Ong, J. Phys. Chem. $B, 2005,109,11100-11109$.

67. Mohammadali Tabatabaei, Alexandre Sangar, Nastaran Kazemi-Zanjani, Philippe Torchio, Alexandre Merlen, and François Lagugné-Labarthet, J. Phys. Chem. C, 2013, 117, $14778-14786$.

68. Shuo Han, Zhibiao Hao,a_Jian Wang, and Yi Luo, J. Vac. Sci. Technol. B, 2005, 23, 15851589.

69. D. F. Liu, Y. J. Xiang, X. C. Wu, Z. X. Zhang, L. F. Liu, L. Song, X. W. Zhao, S. D. Luo, W. J. Ma, J. Shen, W. Y. Zhou, G. Wang, C. Y. Wang, and S. S. Xie, Nano Letters, 2006, 6, 2375-2378.

70. Jea-Young Choi, T. L. Alford, and Christiana B. Honsberg, Langmuir, 2015, 31, 4018-4023.

71. Liang Li, Tianyou Zhai, Haibo Zeng, Xiaosheng Fang, Yoshio Bando and Dmitri Golberg, $J$. Mater. Chem., 2011, 21, 40-56.

72. Paul L. Stiles, Jon A. Dieringer, Nilam C. Shah, and Richard P. Van Duyne, Annu. Rev. Anal. Chem., 2008.

73. Joel Henzie, Jeremy E. Barton, Christopher L. Stender, and Teri W. Odom, Acc. Chem. Res. 2006, 39, 249-257.

74. Rosemary Feeney and Samuel P. Kounaves, Anal. Chem. 2000, 72, 2222-2228.

75. Olga Ordeig, Craig E. Banks, Javier del Campo, Francesc Xavier Munoz, Richard G. Compton, Electroanalysis, 2006, 18, 573- 578.

76. Sebastian J. Hood, Dimitrios. K. Kampouris, Rashid O. Kadara, Norman Jenkinson, 
F. Javier del Campo, Francesc Xavier Munoz and Craig E. Banks, Analyst, 2009, 134, 2301-2305.

77. Jessica Koehne, Jun Li, Alan M. Cassell, Hua Chen, Qi Ye, Hou Tee Ng, Jie Han and M. Meyyappan, J. Mater.Chem., 2004, 14, 676-684.

78. Jun Li, Hou Tee Ng, Alan Cassell, Wendy Fan, Hua Chen, Qi Ye, Jessica Koehne, Jie Han, and M. Meyyappan, Nano Letters, 2003, 3, 597-602.

79. HeaYeon Lee, JongWan Park, JonMin Kim, HoSup Jung, Tomojo Kawai, Applied Physics Letters, 2006, 89, 113901.

80. Napat Triroj, Papot Jaroenapibalb, Haibin Shic, Joanne I. Yehc, Roderic Beresforda, Biosensors and Bioelectronics, 2011, 26, 2927-2933.

81. Zhang XJ, Zhang WM, Zhou XY, Ogorevc B. Anal. Chem. 1996, 68, 3338-3343.

82. Antony S. Dimitrov and Kuniaki Nagayama, Langmuir, 1996, 12, 1303-1311.

83. https://www.inrf.uci.edu/wordpress/wp-content/uploads/sop-wet-nickel-etch.pdf

84. Lee M. Fischer, Maria Tenje, Arto R. Heiskanen, Noriyuki Masuda, Jaime Castillo, Anders Bentien, Jenny Émneus, Mogens H. Jakobsen, Anja Boisen, Microelectronic Engineering, 2009, 86, 1282-1285.

85. N. Aristov, A. Habekost, World J. Chem. Ed. 2015, 3, 115-119.

86. Nicholson, R S., Shain, I, Adv. Anal. Chem., 1964, 36, 706-723.

87. Mairi E. Sandison and Jonathan M. Cooper, Lab on Chip, 2006, 6, 1020-1025.

88. C. Belmonta, M.-L. Terciera, J. Buffle, G.C. Fiaccabrinob, M. Koudelka-Hep, Analytica Chimica Acta, 1996, 329, 203-2 14.

89. Mora F, Segovia G, del Arco A, de Blas M, Garrido P (2012) Stress, neurotransmitters, corticosterone and body-brain integration. Brain Res.

90. Robinson DL, Venton BJ, Heien MLAV, Wightman RM, Clin. Chem., 2003, 49, 1763-1773. 
91. Hefco V, Yamada K, Hefco A, Hrticu L, Tiron A, Nabeshima T, Eur. J. Pharmacol., 2003, 475, 55-60

92. Swamy BEK, Venton BJ, Analyst, 2007, 132, 876-884.

93. Lupu S, Lete C, Marin M, Totir N, Balaure PC, Electrochim Acta, 2009, 54,1932- 1938.

94. Zhao Y, Gao Y, Zhan D, Liu H, Zhao Q, Kou Y, Shao Y, Li M, Zhuang Q, Zhu Z, Talanta, 2005, 66, 51-577.

95. Chernyshov DV, Shvedene NV, Antiova ER, Pletnev IV, Anal Chim Acta, 2008, 621, 178184.

96. Galvan A, Wichmann T, Clin. Neurophysiol., 2008, 119, 1459-1474.

97. Swanson JM, Volkov ND, J Child Psychol Psychiatry, 2009 50, 180-193.

98. Kollins SH, March JS, Biol Psychiatry, 2007, 62, 951-953.

99. Dalley JW, Roiser JP, Dopamine, serotonin and impulsivity. 2012.

100. Carlsson A, Lindqvist M, Magnusson T., Nature, 1957 180, 1200.

101. N. Clausius, C. Born, H. Grunze, Neuropsychiatrie, 2009, 23, 15.

102. K.C. Berridge, T.E. Robinson, J.W. Aldridge, Current Opinion in Pharmacology, 2009, 9, 65 .

103. H. Gunduz-Bruce, Brain Research Reviews, 2009, 60, 279.

104. S.J.G. Lewis, R.A. Barker, Journal of Clinical Neuroscience, 2009, 16, 620.

105. Mark R. Whightman, Science, 2006, 311, 1570-1574.

106. Can Dincera, Rabih Ktaicha, Elmar Laubendera, J. Jakob Heesc, Jochen Kieningerd, Christoph E. Nebelc, Jürgen Heinzea, Gerald A. Urban, Electrochimica Acta, 2015, 185, 101-106. 
107. Zhao Guo-hua, Li Ming-fang, Li Ming-li, Central European Journal of Chemistry, 2007, 5, 1114-1123.

108. Zhen-Huan Shenga,b, Xiao-Qing Zhenga, Jian-Yun Xua, Wen-Jing Baoa, Feng-Bin Wanga, Xing-Hua Xia, Biosensors and Bioelectronics, 2012, 34, 125- 131.

109. Teo Peik-See, Alagarsamy Pandikumar, Huang Nay-Ming, Lim Hong-Ngee and Yusran Sulaiman, Sensors, 2014, 14, 15227-15243.

110. Ali A. Ensafi, M. Taei, Taghi Khayamian, Int. J. Electrochem. Sci., 2010, 5, 116 - 130

111. R. R. Kamatha, and M. J. Madou, ECS Transactions, 2014, 61, 65-73.

112. C. Retna Raj, Takeyoshi Okajima, Takeo Ohsaka, Journal of Electroanalytical Chemistry, 2003, 543, 127-133.

113. P. Tyagi, D. Postetter, D. L. Saragnese, C. L. Randall, M. A. Mirski, and D. H. Gracias, Anal. Chem. 2009, 81, 9979-9984.

114. Hua-Jun Qiu, Gui-Ping Zhou, Guang-Lei Ji, Yun Zhang, Xi-Rong Huang, Yi Ding, Colloids and Surfaces B: Biointerfaces, 2009, 69, 105-108.

115. Reza Karimi Shervedani, Akbar Amini, Bioelectrochemistry, 2012, 84, 25-31.

116. Ping Zhang, Fang-Hui Wu, Guang-Chao ZhaoT, Xian-Wen Wei, Bioelectrochemistry, 2005, 67, 109- 114 .

117. Rajendra Nath Goyal, Vinod Kumar Gupta, Neeta Bachheti, Ram Avatar Sharma, Electroanalysis, 2008, 20, $757-764$.

118. Lei Zhang, Jianbo Jia, Xiangqin Zou, Shaojun Dong, Electroanalysis, 2004, 16, 1413-1418.

119. Blake J. Plowman, Manika Mahajan, Anthony P. O'Mullane,1, Suresh K. Bhargava, Electrochimica Acta, 2010, 55, 8953-8959. 
120. Chia-Liang Suna, Hsin-Hsien Leea, Jen-Ming Yanga, Ching-Chou Wub, Biosensors and Bioelectronics, 2011, 26, 3450-3455.

121. Michael L. A. V. Heien, Amina S. Khan, Jennifer L. Ariansen, Joseph F. Cheer, Paul E. M. Phillips, Kate M. Wassum, and R. Mark Wightman, PNAS , 2005, 102, 10023-10028.

122. Feng Zhua, Jiawei Yana, Miao Lub, Yongliang Zhoua, Yang Yanga, Bingwei Maoa, Electrochimica Acta, 2011, 56, 8101-8107.

123. Anupama Aggarwal, Mengjia Hu \& Ingrid Fritsch, Anal Bioanal Chem, 2013, 405, 3859 3869.

124. Bhavik Anil Patel, Collin C. Luk, Pei Ling Leow, Arthur J. Lee, Wali Zaidic and Naweed I. Syed, Analyst, 2013, 138, 2833.

125. Chaoxiong Ma, Nicholas M. Contento, Larry R. Gibson, II, and Paul W. Bohn, Anal. Chem., 2013, 85, 9882-9888.

126. Matthew K. Zachek, Andre Hermans, R. Mark Wightman, and Gregory S. McCarty, $J$ Electroanal Chem, 2008, 614, 113-120. 